\title{
PARTICULARITIES OF THE AQUATIC VEGETATION FROM “IRON GATES” NATURA 2000 SITE (BANAT, ROMANIA)
}

\author{
Irina GOIA * and Adrian OPREA ** \\ * "Babeş-Bolyai” University of Cluj-Napoca, Faculty of Biology and Geology, Gheorghe Bilaşcu Street \\ 44, Romania, Cluj-Napoca, Cluj County, RO-400006, irina.goia@ubbcluj.ro \\ ** "Anastasie Fatu" Botanic Garden of Iaşi, University "Alexandru Ioan Cuza”, Dumbrava Roşie Sreet \\ 7-9, Iaşi, Iaşi County, Romania, RO-700487, aoprea@uaic.ro, a_aoprea@yahoo.co.uk
}

DOI: 10.1515/trser-2015-0035

KEYWORDS: Romania, Danube River, “Iron Gates”, aquatic vegetation, natural habitats.

\section{ABSTRACT}

The paper presents the results of investigations on the aquatic vegetation, along the Romanian bank of the Danube River, in the area of Porţile de Fier ("Iron Gates"; Mehedinţi and Caraş-Severin counties), a Natura 2000 site. Twenty-three plant communities were identified from Lemnetea minoris and Potametea pectinati classes. The survey led to the identification of some newly described phytocoenotaxons in this protected area. All the plant communities in this paper are documented by phytosociologic tables, being accompanied by coenotaxonomic, phytogeographical, ecological and social strategies analysis, in order to assess their conservation status, as the main tool for management decisions.

ZUSAMMENFASSUNG: Die Besonderheiten der Gewässervegetation im Natura 2000-Gebiet „Porțile de Fier/Eisernes Tor”.

Vorliegende Arbeit stellt die Ergebnisse der Untersuchungen betreffend die Gewässervegetation entlang der rumänischen Uferstrecke der Donau im Bereich des Natura 2000-Gebietes „Porţile de Fier/Eisernes Tor” (Verwaltungskreise Mehedinţi und CaraşSeverin) vor. Dabei wurden 23 Pflanzengesellschaften der Klassen Lemnetea minoris und Potametea pectinati festgestellt. Die Untersuchungen führten zur Identifikation und Beschreibung einiger für dieses Schutzgebiet neuer phytocoenologischer Einheiten. Alle in der Arbeit vorgestellten Pflanzengesellschaften sind mittels pflanzensoziologischer Tabellen dokumentiert und von coenotaxonomischen, pflanzengeographischen, ökologischen sowie sozial-strategischen Analysen begleitet, die der Bewertung des Erhaltungszustandes als wichtigstes Instrument für Management-Entscheidungen dienen.

REZUMAT: Particularităţile vegetaţiei acvatice din situl Natura 2000 „Porţile de Fier” (Banat, România).

Lucrarea prezintă rezultatele investigaţiilor efectuate asupra vegetaţiei acvatice de-a lungul malului românesc al Dunării, în zona sitului Natura 2000 Porţile de Fier (jud. Mehedinţi şi Caraş-Severin). Au fost identificate 23 asociaţii vegetale aparţinând claselor Lemnetea minoris şi Potamogetonetea (syn. Potametea) pectinati. Studiile au condus la identificarea unor fitocenotaxoni noi pentru aria protejată. Toate asociaţiile prezentate în lucrare sunt documentate prin tabele fitosociologice, fiind însoţite şi de analize cenotaxonomice, fitogeografice, ecologice şi ale strategiilor sociale, în scopul evaluării stării de conservare a acestora, ca principal instrument în luarea deciziilor manageriale. 


\section{INTRODUCTION}

The complexity of aquatic communities is a result of topographic diversity (VivianSmith, 1997), of physical and chemical characteristics of the water and of human influences (Tetelea, 2005; Brönmark and Hansson, 2010).

Species diversity is dependent on habitat structure, the most complex habitats hosting a higher biodiversity (Bell et al., 1991). According to Williams et al. (2003), pounds are responsible for a higher diversity comparing to other aquatic communities, sheltering unique and rare species. Aquatic macrophytes play an important role for nitrogen (Saunders and Kalff, 2001) and detritus retention (Rooke, 1984), during the vegetation season. Moreover, higher structural complexity of habitats provides higher heterogeneity of algae and invertebrates colonization (Dudley, 1988; Melo et al., 2002; Takeda et al., 2003; Thorp et al., 1997).

A complex system of rivers, ponds, ditches, swamps, and flooded areas were formed as a result of the construction of one of the largest hydroelectric power plants in Europe, "Iron Gates” I, on the Danube River, in 1972. Both, the isle of Moldova Veche and Nera Delta are large areas flooded during the spring season. According to Blaustein and Schwartz, 2001 (in Dudley), temporary water host important species for global biodiversity and plays an important role for population dynamics and community structure studies.

The "Porţile de Fier" Natural Park was the subject of many botanical surveys, addressing all the plant communities, a highly diversified area, leading to a floristic inventory of 1,875 vascular plant species: among these 1,748 species, 120 subspecies and six varieties. Also, there have been registered 44 plant communities, framed in two suballiances, 22 alliances, 16 orders, and 13 classes of vegetation (Matacă, 2005). Aquatic and wetlands flora found along the Danube River, in the "Iron Gates" Natural Park, has been studied since the nineteenth century, by Grecescu (1898), Heuffel (1858), but especially in the last century (Borza, 1947-1949; Matacă, 2002; Roman, 1971; Călinescu and Iana, 1964; Raclaru and Alexan, 1972; Ciocârlan et al., 1969; Liţescu et al., 2003; Morariu et al., 1969; Sârbu et al., 2006, 2011; Ştefureac et al., 1971).

As a result of the newly designed navigation system in the "Porţile de Fier" area, the vegetation investigations increased (Matacă, 2002; Ştefureac, 1970; Grigore and Coste, 1978; Raclaru and Alexan, 1973; Resmeriţă et al., 1972; Şerbănescu and Sanda, 1970; Csürös et al., 1968; Dihoru et al., 1973; Păun et al., 1968; Sanda et al., 1968, 1970; Todor et al., 1971).

The purpose of this study is a reappraisal of the previously identified aquatic communities, new plant communities in the area, and the analysis of their structure, primarily to highlight the conservation status of habitats, emphasizing the presence of invasive plant species. It was taken into account the fishermen complaint toward the explosive growth of fixed aquatic vegetation, especially those communities edified by the water chestnut (Trapa natans). This species is known as having a wide ecological spectrum, concerning nutrient and heavy metal concentration (Rai and Sinha, 2001), playing an important role in phytoremediation (Srivastava et al., 2014).

The surveys have been conducted mainly in the Natura 2000 site "ROSPA0026 Danube sector - Baziaş-Porţile de Fier", largely overlapping the "Iron Gates" Natural Park. The above mentioned site has an area of 9,904 ha, and is located on the territory of CaraşSeverin (56\%) and Mehedinţi (44\%) counties (south-western part of Romania), in Continental biogeographical region. The altitude ranges from 28 to $192 \mathrm{~m}$ a.s.l. (O. M. 2387/2001; Planul de management al Parcului Natural Porţile de Fier, 2013) (Fig. 1). 


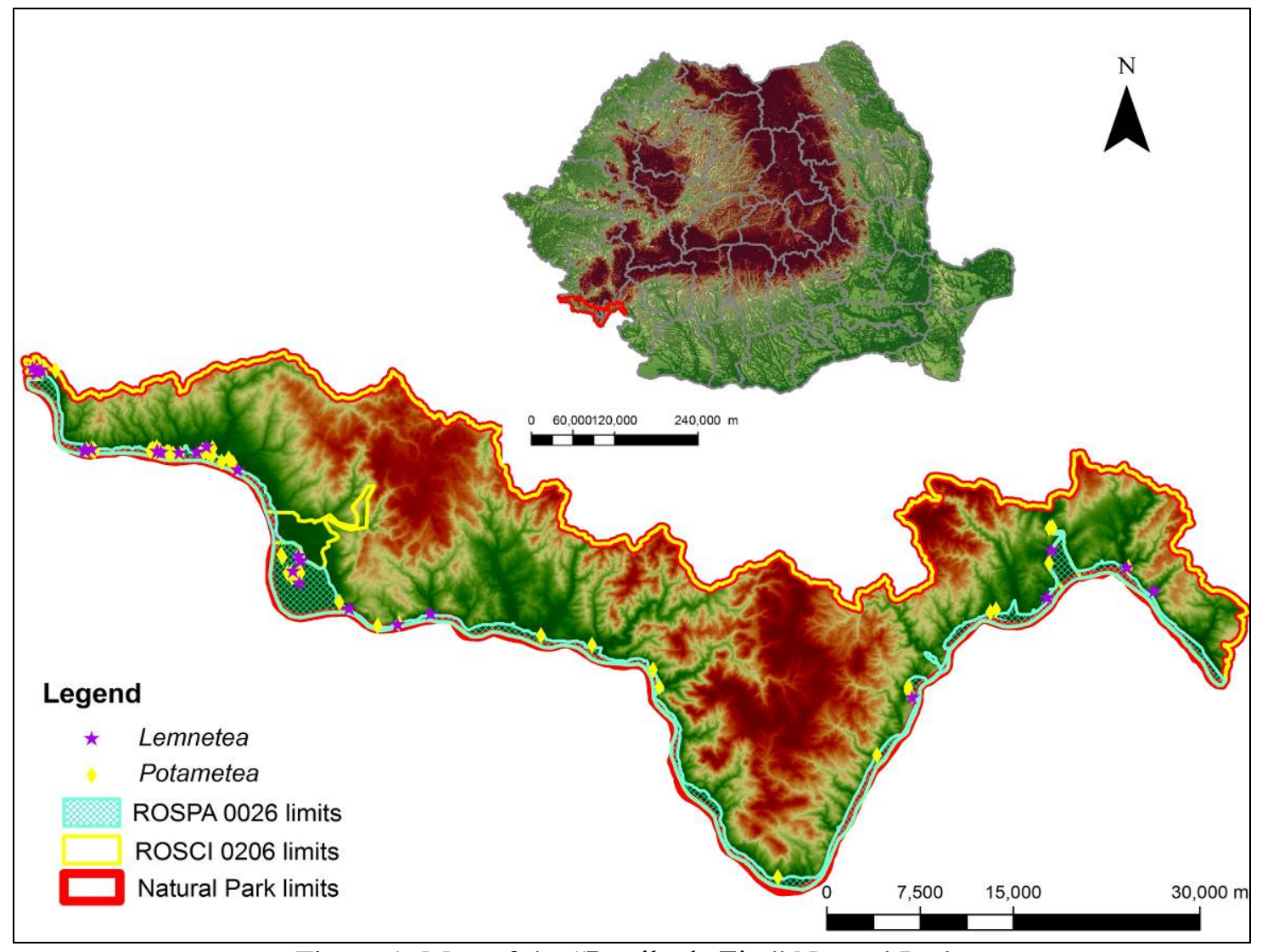

Figure 1: Map of the "Porţile de Fier” Natural Park.

Geology of the study area has a mosaic feature. The studied territory is the most picturesque section of the Danube River - the so-called areas "Cazanele Mari” and "Cazanele Mici". In addition to these, from a geological point of view there is the remarkable suspended synclinal called "Munteana", the fossiliferous outcrops at Sviniţa and Bahna, the Permian volcanic neck Trescovăţ, and also a variety of karst formations. In some areas, the loess deposits are exposed in the form of slopes, some including a status of nature reserves, for example "Râpa cu Lăstuni" (a nesting place for some species of swifts). The Moldova Veche isle was intended to become into a place to store the mining waste from the MoldaMin Company, which exploited copper in Moldova Nouă, and coal at Cozla, Baia Nouă and Bigăr.

The surveyed territory belongs to the "Iron Gates" Massif (Popp, 1971), which is crossed for $134 \mathrm{~km}$ by the Danube River, generating the most spectacular gorge sector in Europe (Călinescu et al., 1955; The Geological Map of Romania). Upon entering Romania, the riverbed is between 7.5 to $17.5 \mathrm{~m}$ in depth and has a width of up to $1,500 \mathrm{~m}$. Between Coronini and Liuborajdea, the Danube passes through a limestone sector, the width decreases and reaches $36 \mathrm{~m}$ in depth. West of Liubcova, the Danube crosses a granite area, and toward Cozla rapids and transverse currents occur, due to substrate formed by gneisses of Ielova, conglomerates, porphyry and porphyrites. Downstream Cozla, the Danube cuts through another limestone area. The maximum depth is recorded in the "Cazane" area - about $100 \mathrm{~m}$, and the width is 150-200 m. Beyond the "Cazane" area, the riverbed depth decreases and because of the lithology, represented by crystalline schists, rapids and submerged rocks appear. 
Based on the calculated values for the period during 1970-2000, average annual temperatures in the "Iron Gates" Natural Park are: at Berzasca $11.4^{\circ} \mathrm{C}$, at Orşova $11.6^{\circ} \mathrm{C}$, at Sviniţa $11.5^{\circ} \mathrm{C}$; the relative humidity of the air at Orşova is $76 \%$ and at Drobeta-Turnu Severin is of $74 \%$. There were two periods recorded with the most rainfall, one in May-June and the second in November-December. At Orşova, the average yearly rainfall is of $586.3 \mathrm{~mm}$, and at Drobeta-Turnu Severin is of $652.4 \mathrm{~mm}$. The prevailing winds at Orşova are from the northwest, and at Drobeta-Turnu Severin those from west and north-west (Matacă, 2005).

From a pedologic point of view, the most widespread zonal soils are clay-illuvial podzolic series, the brown-acid soils, and intrazonal lito-morphic soils. The best soils that are presented in flooded areas are those weakly evolved soils, namely silt and alluvial soils, that vary in texture and evolution. On the high river meadow and lower terrace there is a succession of alluvial soils: sandy silt layered alluvials, the alluvial humiferous soils, and the brown alluvial soils. On alluvial cones and tributaries floodplains, soils are coarse, and in the calacareous skeleton occur branciogs. In some places, alluvial gleic soils and even swamps gleic soils are reported (Matacă, 2005; Glăvan and Geanana, 1972).

\section{MATERIAL AND METHODS}

The study of vegetation utilizes a basic coenotaxonomic unit the plant association, characterized into the field by the phytocoenoses (= individuals of association), which were analyzed on the basis of phytocenological relevés. The quantitative indices of abundancedominance (AD) used are those from the Braun-Blanquet scale (Braun-Blanquet, 1964). Identification of plant associations was based on the characteristic species, taking into account the presence of the dominant species (Cristea, 1991, 1993; Cristea et al., 2004). In order to draw up the coenotaxonomic conspectus, various records have been consulted (Coldea, 1991, 1997; Grigore and Coste, 1978; Morariu and Danciu, 1970; Popescu and Ştefureac, 1976; Raclaru and Alexan, 1973; Şerbănescu and Sanda, 1970; Dihoru et al., 1973; Morariu et al., 1969; Popescu et al., 1997; Sanda et al., 1968, 1980, 1994; Todor et al., 1971), as well as other available European classifications.

The extent of the sampled surface was determined by the size of the phytocoenoses, ranging from $1 \mathrm{~m}^{2}$ to $25 \mathrm{~m}^{2}$.

Setting the location of the natural habitats were based on the characteristic phytocoenotaxons (associations, alliances, orders), as stated by the Romanian interpretation manual of habitats (Gafta and Mountford, 2008; Doniţă et al., 2005, 2006), in accordance with the European Union Habitat Directive 92/43/EEC (***, Manuel d'interpretation des habitats de l'UE).

The vascular flora nomenclature is in accordance with Flora Europaea (Tutin et al., 1964-1980; ***, http://ww2.bgbm.org/europlusmed/), and with some Romanian identification field books (Ciocârlan, 2000). We used the nomenclature of the algae suggested by Cărăuş (2012). Establishing the values of ecologic and floristic elements indexes were made after various reference publications (e.g. Popescu and Sanda, 1998; Sanda et al., 1983). The social behaviour and the degree of naturalness were analyzed after Borhidi (1995).

The coenotaxonomic classification was performed using the program SYNTAX 5.0 (Podani, 1993), using the UPGMA algorithm and also the Bray Curtis quantitative index. 


\section{RESULTS AND DISCUSSION}

Twenty-three aquatic vegetal communities were identified in the area, all belonging in two classes, according to the following coenotaxonomic conspectus:

${ }^{*}$ new recorded associations in the studied area

\section{LEMNETEA O. de Bolós and Masclans 1955}

Lemnetalia minoris O. de Bolós and Masclans 1955

Lemnion gibbae R. Tx. and Schwabe-Braun in R. Tx 1974

1. Lemnetum gibbae Miyavaki and J. Tüxen 1960

2. Lemnetum minoris Oberd. ex T. Müller and Görs 1960

3. Lemno-Spirodeletum W. Koch 1954 (syn. Spirodeletum polyrhizae W. Koch 1954)

4. Salvinio-Spirodeletum polyrhizae Slavnič 1956

5. *Lemno-Salvinietum natantis Myawaki and Tx. 1960

6. * Lemno minoris-Azolletum filiculoides Br.-Bl. 1952

Lemno-Utricularietalia Passarge 1978

Utricularion vulgaris Passarge 1964

7. " Lemno-Utriculariteum vulgaris Soó (1928) 1947

Hydrocharietalia Rübel 1933

Hydrocharition morsus-ranae (Passarge 1964) Westhoff and den Held 1969

8. Hydrocharitetum morsus-ranae Van Langendonk 1935

9. Ceratophylletum demersi Corillion 1957

POTAMETEA PECTINATI R. Klika in Klika and Novák 1941

Potametalia pectinati W. Koch 1926

Potamion pectinati (W. Koch 1926) Görs 1977

10. *Potametum lucentis Hueck 1931

11. Myriophyllo-Potametum lucentis Soó 1934 -*vallisnerietosum (syn. Potameto-Vallisnerietum Br.-B1 1931)

12. *Najadetum marinae Fukarek 1961

13. *Najadetum minoris Ubrizsy 1961

14. *Potametum pectinati (Hueck 1931) Carstensen 1955

15. Potametum pusilli Soó 1927

16. *Elodeetum nuttallii Ciocârlan et al. 1997

17. "Elodeetum canadensis (Pign. 1953) Pass. 1964

Nymphaeion albae Oberd. 1957

18. Nymphoidetum peltatae (All. 1922) Bellot 1951

19. Trapetum natantis Kárpáti 1963

20. Potametum natantis Soó 1927

21. *Potametum perfoliati Miljan 1933

Ranunculion aquatilis Passarge 1964

22. *Ranunculetum (syn. Batrachietum) trichophylli Soó (1927) 1971

23. Potametum nodosi Passarge 1964.

Class Lemnetea O. de Bolós and Masclans 1955 in the "Porţile de Fier" Natural Park is represented by nine plant communities, belonging to three orders and three alliances (Tab. 1). 
Table 1: Plant communities from Class Lemnetea O. de Bolós and Masclans 1955.

\begin{tabular}{|c|c|c|c|c|c|c|c|c|c|c|}
\hline Relevé no. & 1 & 2 & 3 & 4 & 5 & 6 & 7 & 8 & 9 & 10 \\
\hline General coverage (\%) & 70 & 90 & 80 & 100 & 100 & 100 & 100 & 90 & 80 & 100 \\
\hline Relevé area $\left(\mathrm{m}^{2}\right)$ & 9 & 9 & 4 & 9 & 2 & 1 & 5 & 9 & 1 & 25 \\
\hline Relevé code & $\begin{array}{l}\stackrel{8}{R} \\
\text { 自 } \\
g\end{array}$ & $\begin{array}{l}\mathscr{m}_{1} \\
\dot{B}_{1} \\
\boldsymbol{G}_{1}\end{array}$ & 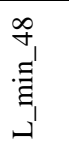 & 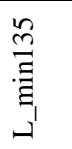 & 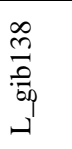 & 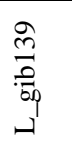 & 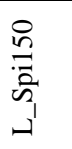 & $\begin{array}{l}\hat{m} \\
\overrightarrow{\tilde{n}} \\
\tilde{\omega}_{1} \\
\omega^{\prime}\end{array}$ & 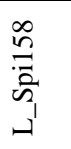 & $\begin{array}{l}\tilde{B} \\
= \\
\tilde{\tilde{n}} \\
\omega^{\prime}\end{array}$ \\
\hline \multicolumn{11}{|l|}{ Lemnion } \\
\hline Lemna gibba & . & . & . & 1 & 3 & $3-4$ & + & . & 1 & . \\
\hline Spirodela polyrhiza & + & +-1 & + & 1 & + & 2 & 5 & 4 & 2 & $1-2$ \\
\hline \multicolumn{11}{|l|}{ Hydrocharition } \\
\hline $\begin{array}{l}\text { Hydrocharis morsus- } \\
\text { ranae }\end{array}$ & . & . & . & . & . & - & . & . & . & . \\
\hline \multicolumn{11}{|l|}{ Utricularion } \\
\hline Utricularia vulgaris & . & . & . & . & . & . & . & . & . & . \\
\hline \multicolumn{11}{|l|}{$\begin{array}{l}\text { Lemnetalia and } \\
\text { Lemnetea }\end{array}$} \\
\hline Azolla filiculoides & . & . & . & . & + & 1 & . & . & 1 & + \\
\hline Lemna minor & 4 & $4-5$ & 4 & 5 & $3-4$ & 3 & $1-2$ & $2-3$ & 3 & 3-4 \\
\hline Salvinia natans & . & . &. &. & . & . & + & + & + & + \\
\hline \multicolumn{11}{|l|}{ Potamion } \\
\hline Potamogeton acutifolius & . & . & . & . & . & . & . & . & . & . \\
\hline $\begin{array}{l}\text { Potamogeton acutifolius } \\
\text { f. major }\end{array}$ & . & . & - & . & . & . & . & . & . & . \\
\hline \multicolumn{11}{|l|}{$\begin{array}{l}\text { Potametalia and } \\
\text { Potametea }\end{array}$} \\
\hline Najas marina & . & . & . & . & . & . & . & . & . & . \\
\hline Najas minor & . & . & . & . & . & . & . & . & . & . \\
\hline Potamogeton nodosus & . & 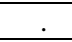 & . & . & . & . & . & . & . & . \\
\hline Trapa natans & 1 & + & . & . & . & . & . & . & . & + \\
\hline $\begin{array}{l}\text { Ceratophyllum } \\
\text { demersum }\end{array}$ & . & . & . & . & . & . & . & . & . & 1 \\
\hline Elodea canadensis & . & . & . & . & . & . & . & . & . & . \\
\hline Myriophyllum spicatum & . & . & . & . & . & . & . & . & . & . \\
\hline Potamogeton crispus & . & . & . & . & . & . & . & . & . & + \\
\hline Potamogeton natans & 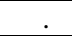 & 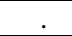 & . & . & . & . & . & . & . & . \\
\hline Potamogeton pectinatus & 1 & 2 & . & . & . & . & . & . & . & . \\
\hline $\begin{array}{l}\text { Potamogeton } \\
\text { perfoliatus }\end{array}$ & . & r & . & - & . & . & . & . & . & + \\
\hline Potamogeton pusillus & . & . & . & . & . & . & . & . & . & . \\
\hline \multicolumn{11}{|l|}{ Elodea nuttallii } \\
\hline \multicolumn{11}{|l|}{$\begin{array}{l}\text { Phragmiti- } \\
\text { Magnocaricetea }\end{array}$} \\
\hline $\begin{array}{l}\text { Alisma plantago- } \\
\text { aquatica }\end{array}$ & . & . & . & . & . & . & . & . & . & . \\
\hline $\begin{array}{l}\text { Schoenoplectus } \\
\text { lacustris }\end{array}$ & . & . & . & . & . & . & . & . & . & . \\
\hline Paspalum distichum & . & . & . & . & . & . & . & . & . & . \\
\hline \multicolumn{11}{|l|}{ Varyae syntaxa } \\
\hline Echinochloa crus-galli & . & . & . & . & . & . & . & . & . & . \\
\hline Cladophora glomerata & . &. & 2 & . & . & & . &. & 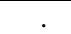 & + \\
\hline
\end{tabular}


Table 1 (continued): Plant communities from Class Lemnetea O. de Bolós and Masclans 1955.

\begin{tabular}{|c|c|c|c|c|c|c|c|c|c|c|}
\hline Relevé no. & 11 & 12 & 13 & 14 & 15 & 16 & 17 & 18 & 19 & 20 \\
\hline General coverage (\%) & 100 & 80 & 85 & 100 & 100 & 90 & 98 & 90 & 90 & 100 \\
\hline Relevé area $\left(\mathrm{m}^{2}\right)$ & 2 & 9 & 9 & 9 & 9 & 9 & 9 & 1 & 9 & 9 \\
\hline Relevé code & 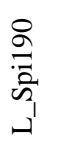 & 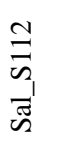 & 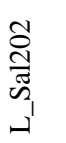 & $\begin{array}{l}\stackrel{8}{0} \\
\text { त्ञ } \\
\tilde{n}_{1} \\
\lrcorner^{\prime}\end{array}$ & 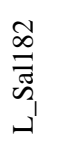 & $\begin{array}{l}\vec{\infty} \\
\vec{\Xi} \\
\tilde{\omega}_{1} \\
.\end{array}$ & 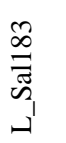 & 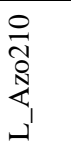 & 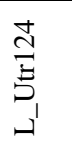 & 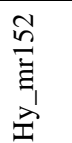 \\
\hline \multicolumn{11}{|l|}{ Lemnion } \\
\hline Lemna gibba & + & . & . & . & . & . & . & . & . & . \\
\hline Spirodela polyrhiza & $2-3$ & 2 & . & +-1 & +-1 & + & + & + & . & + \\
\hline \multicolumn{11}{|l|}{ Hydrocharition } \\
\hline $\begin{array}{l}\text { Hydrocharis morsus- } \\
\text { ranae }\end{array}$ & . & . & . & . & . & . & . & . & . & $3-4$ \\
\hline \multicolumn{11}{|l|}{ Utricularion } \\
\hline Utricularia vulgaris & . & . & . & . & . & . & . & . & 5 & 1 \\
\hline \multicolumn{11}{|l|}{$\begin{array}{l}\text { Lemnetalia and } \\
\text { Lemnetea }\end{array}$} \\
\hline Azolla filiculoides & + & . & $1-2$ & $1-2$ & $+\ldots 1$ & $1-2$ & $1-2$ & $3-4$ & . & . \\
\hline Lemna minor & $3-4$ & $1-2$ & + & $1-2$ & + & $1-2$ & + & 2 & + & . \\
\hline Salvinia natans & 1 & $3-4$ & 4 & $3-4$ & $3-4$ & 4 & $4-5$ & $1-2$ & . & + \\
\hline \multicolumn{11}{|l|}{ Potamion } \\
\hline Potamogeton acutifolius & . & . & . & . & . & . & . & . & . & . \\
\hline $\begin{array}{l}\text { Potamogeton acutifolius } \\
\text { f. major }\end{array}$ & $\cdot$ & . & . & . & . & . & . & . & . & . \\
\hline \multicolumn{11}{|l|}{$\begin{array}{l}\text { Potametalia and } \\
\text { Potametea }\end{array}$} \\
\hline Najas marina & & . & + & . & . & . & . & . & . & . \\
\hline Najas minor & . & . & . & . & . & . & . & . & . & . \\
\hline Potamogeton nodosus & & . & 1 & . & . & . & . & . & . & . \\
\hline Trapa natans & 1 & . & . & . & $+\ldots 1$ & . & $1-2$ & . & . & . \\
\hline Ceratophyllum demersum & . & . & . & . & . & . & . & . & . & $2-3$ \\
\hline Elodea canadensis & . & . & . & . & . & . & $1-2$ & . & . & 4 \\
\hline Myriophyllum spicatum & . & . & . & . & . & . & . & . & . & . \\
\hline Potamogeton crispus & . & . & . & . & . & . & . & . & . & . \\
\hline Potamogeton natans & . & . & . & . & . & . & . & . & + & . \\
\hline Potamogeton pectinatus & . & . & . & . & . & . & . & . & . & . \\
\hline Potamogeton perfoliatus & . & . & . & . & . & . & . & . & . & . \\
\hline Potamogeton pusillus & . & . & . & . & . & . & . & . & . & . \\
\hline Elodea nuttallii & 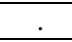 & . & 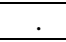 & 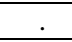 & . & . & . & . & . & . \\
\hline \multicolumn{11}{|l|}{$\begin{array}{l}\text { Phragmiti- } \\
\text { Magnocaricetea }\end{array}$} \\
\hline $\begin{array}{l}\text { Alisma plantago- } \\
\text { aquatica }\end{array}$ & . & . & . & . & . & . & . & . & 1 & . \\
\hline Schoenoplectus lacustris & . & . & . & . & . & . & . & . & . &. \\
\hline Paspalum distichum & & 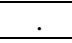 & - & 3 & 3 & . & . & $1-2$ & & . \\
\hline \multicolumn{11}{|l|}{ Varyae syntaxa } \\
\hline Echinochloa crus-galli & 2 & . & . & . & . & . & . & . & . & . \\
\hline Cladophora glomerata & & 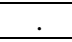 & - & - & 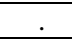 & - & - & . & & - \\
\hline
\end{tabular}


Table 1 (continued): Plant communities from Class Lemnetea O. de Bolós and Masclans 1955.

\begin{tabular}{|c|c|c|c|c|c|c|c|c|c|c|c|}
\hline Relevé no. & 21 & 22 & 23 & 24 & 25 & 26 & 27 & 28 & 29 & 30 & 31 \\
\hline General coverage (\%) & 60 & 50 & 100 & 100 & 100 & 70 & 85 & 65 & 90 & 90 & 100 \\
\hline Relevé area $\left(\mathrm{m}^{2}\right)$ & 8 & 25 & 25 & 25 & 9 & 9 & 9 & 9 & 25 & 9 & 25 \\
\hline Relevé code & 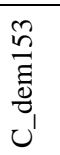 & 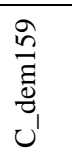 & 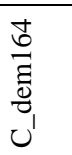 & 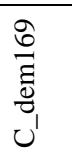 & 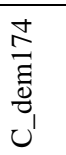 & 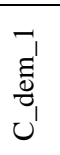 & $\begin{array}{l}\infty \\
\Xi_{U}^{\prime} \\
\Xi_{U}^{\prime}\end{array}$ & 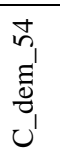 & 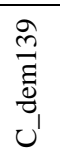 & $\begin{array}{l}\vec{\nabla} \\
\vec{\Xi} \\
\underbrace{\prime}_{0} \\
ن\end{array}$ & 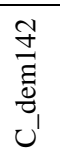 \\
\hline \multicolumn{12}{|l|}{ Lemnion } \\
\hline Lemna gibba & 1 & . & . & . & . & . & . & . & . & . & . \\
\hline Spirodela polyrhiza & $3-4$ & . & $1-2$ & + & $1-2$ & . & . & . & + & + & . \\
\hline \multicolumn{12}{|l|}{ Hydrocharition } \\
\hline $\begin{array}{l}\text { Hydrocharis morsus- } \\
\text { ranae }\end{array}$ & + & . & . & . & . & . & . & . & . & . & . \\
\hline \multicolumn{12}{|l|}{ Utricularion } \\
\hline Utricularia vulgaris & + & . & . & . & . & . & . & . & . & . & . \\
\hline \multicolumn{12}{|l|}{$\begin{array}{l}\text { Lemnetalia and } \\
\text { Lemnetea }\end{array}$} \\
\hline Azolla filiculoides & . & . & + & + & $+\ldots 1$ & . & . & . & . & . & . \\
\hline Lemna minor & . & . & $3-4$ & $1-2$ & $3-4$ & . & . & . & + & . & + \\
\hline Salvinia natans & . & . & + & + & + & . & . & . & . & . & . \\
\hline \multicolumn{12}{|l|}{ Potamion } \\
\hline Potamogeton acutifolius & . & . & 1 & . & . & . & . & . & . & $1-2$ & + \\
\hline Potamogeton a. f. major & . & . & 1 & . & . & . & . & . & . & $1-2$ & + \\
\hline \multicolumn{12}{|l|}{$\begin{array}{l}\text { Potametalia and } \\
\text { Potametea }\end{array}$} \\
\hline Najas marina & . & . & . & + & + & . & . & . & + & 2 & + \\
\hline Najas minor & . & . & . & . & . & $\bar{c}$ & . & . & . & 1 & + \\
\hline Potamogeton nodosus & . & . & 1 & . & . & $1-2$ & . & & . & . & . \\
\hline Trapa natans & . & . & 1 & 1 & + & . & . & . & . & + & 1 \\
\hline Ceratophyllum demersum & 4 & $2-3$ & 4 & 5 & 4 & 3 & 4 & 4 & 5 & 3 & 4 \\
\hline Elodea canadensis & + & . & . & . & $1-2$ & . & . & . & . & . & 2 \\
\hline Myriophyllum spicatum & . & . & . & . & . & . & + & - & . & . & . \\
\hline Potamogeton crispus & . & . & . & . & + & . & . & . & . & . & . \\
\hline Potamogeton natans & . & . & . & . & . & . & . & 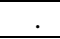 & . & . & . \\
\hline Potamogeton pectinatus & . & . & + & . & . & 1 & . & + & . & . & . \\
\hline Potamogeton perfoliatus & . & 2 & + & + & . & $2-3$ & + & . & . & . & . \\
\hline Potamogeton pusillus & . & . & . & . & . & . & + & + & . & . & . \\
\hline Elodea nuttallii & . & . & . & + & . & . & . & . & . & . & . \\
\hline \multicolumn{12}{|l|}{$\begin{array}{l}\text { Phragmiti- } \\
\text { Magnocaricetea }\end{array}$} \\
\hline $\begin{array}{l}\text { Alisma plantago- } \\
\text { aquatica }\end{array}$ & . & . & . & . & . & . & . & . & . & . & . \\
\hline Schoenoplectus lacustris & . & . & . & . & . & . & . & + & . & . & . \\
\hline Paspalum distichum & . & . & . & . & . & . & . & . & - & . & . \\
\hline \multicolumn{12}{|l|}{ Varyous syntaxa } \\
\hline Echinochloa crus-galli & . & . & . & 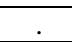 & . & . & . & & . & . & . \\
\hline Cladophora glomerata & . & . & + & + & 1 & 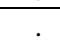 & 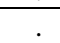 & & & 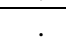 & . \\
\hline
\end{tabular}

Data and place of relevés: Ass. Lemnetum minoris: rel. 1-3, 25.06.2012, Divici Pond, rel. 4, 31.07.2012, Calinovăţ Island; Ass. Lemnetum gibbae: rel. 5-6, 01.08.2012, Calinovăț Island; Ass. Lemno-Spirodeletum: rel. 7, 01.08.2012, Nera Pond, rel. 8, 03.08.2012, Calinovăt Island, rel. 9, 02.08.2012, Nera Pond, rel. 10, 03.08.2012, Liborajdea, rel. 11, 23.09.2012, 
Moldova Veche Island; Ass. Salvinio-Spirodeletum polyrhizae, rel. 12, 27.06.2012, Nera Pond; Ass. Lemno-Salvinietum natantis, rel. 13, 24.09.2012, Pojejena, rel. 14, 24.09.2012, Orşova, rel. 15-17, 23.09.2012, Moldova Veche Island; Ass. Lemno minoris-Azolletum filiculoides: rel. 18, 25.09.2012, Orşova; Ass. Lemno-Utriculariteum vulgaris: rel. 19, 31.07.2012, Moldova Veche Island; Ass. Hydrocharitetum morsus-ranae: rel. 20, 02.08.2012, Nera Pond; Ass. Ceratophylletum demersi: rel. 21, 02.08.2012, Nera Pond, rel. 22, 02.08.2012, Coronini, rel. 23, 03.08.2012, Liborajdea, rel. 24, 03.08.2012, Dubova, rel. 25, 05.08.2012, Orşova, rel. 26, 23.06.2012, Slătinicul Mare Valley, rel. 27, 23.06.2012, Vodiţei Valley, rel. 28, 25.06.2012, Belobreşca Pond, rel. 29, 01.08.2012, Şuşca Pond, rel. 30-31, 01.08.2012, Pojejena.

Three plant communities are reported for the first time in this protected area. Class Lemnetea comprises of aquatic vegetation that is short in size, as well as scattered throughout lakes, ponds, and canals. These communities appear as free floating or initially fixed to the substrate, later roots break and become free, being carried by water currents. This is the reason why in the dendrogram (Fig. 4), communities of this class do not form distinct clusters. In the studied area, this vegetation type has a simple coenotic structure, with a well individualized coenotic core, usually represented by at least $50 \%$ of the characteristic species for class and the coenotaxonomic component units (in Lemnetea gibbae 100\%), the other plant species being characteristic especially for Potametea class, with which is in contact. The vegetation cycle (growing season) is very short, only few weeks.

From a phytogeographical point of view (Fig. 2), the cosmopolitan and Eurasian species are thrive, especially the Mediterranean species, as a result of the climate influence in south-western Romania. In general, the plant communities within this class are poor in species. Ceratophylletum demersi is richer in species, moreover it is the most widespread plant community in the area, which is reflected by a greater variety of phytogeographical elements.

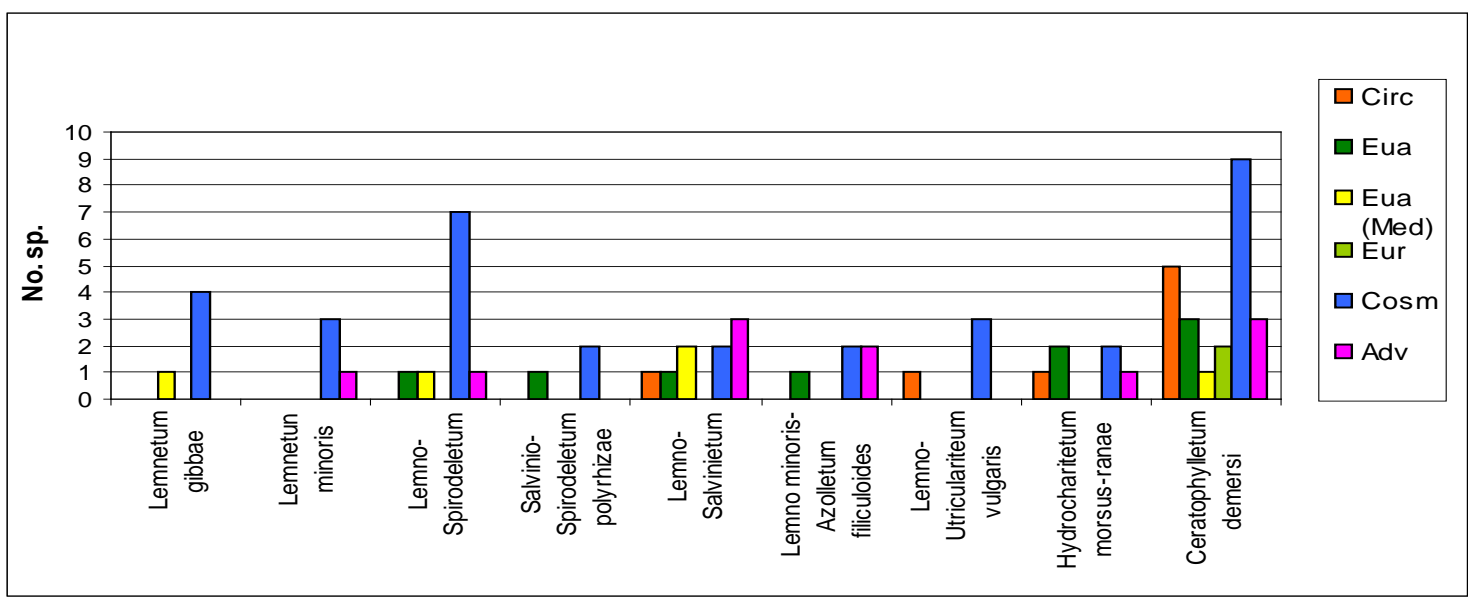

Figure 2: Phytogeographical spectrum for the associations of the Lemnetea class. 


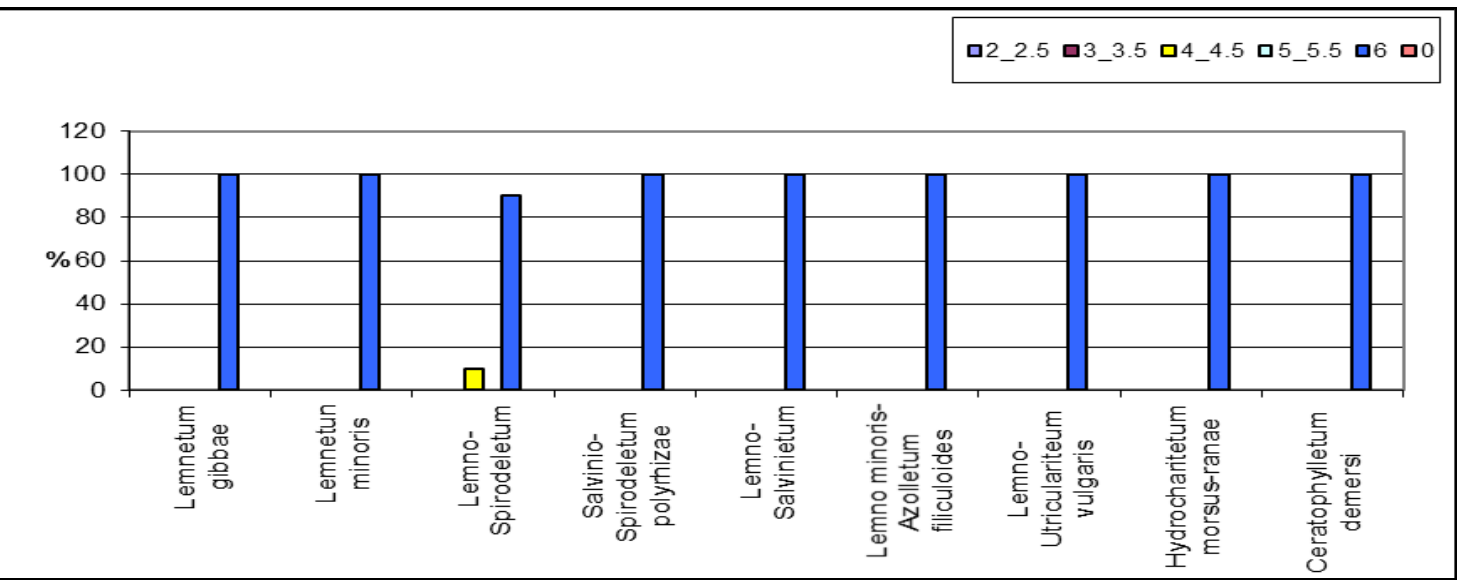

Figure 3: Ecological spectrum for humidity for the communities of the Lemnetea class.

Among the alien plant species in this class, the more common is Azolla filiculoides, on Moldova Veche Isle; along with this species, Paspalum distichum is also present.

The ecological spectrum shows the extreme characteristics of this habitat, 100\% dominated by the hydrophilous plant species $\left(\mathrm{U}_{6}\right)$ (Fig. 3). The temperate climate is reflected by the predominance of micro-mesothermal species $\left(\mathrm{T}_{3-3.5}\right)$, and the influence of the Mediterranean climate is evidenced by the presence of the moderately thermophilous species ( $T_{4-4.5}$ ) and thermophilous ones $\left(T_{5-5.5}\right)$ (Fig. 5). Related to water pH (Fig. 6), the dominant species are those euri-ionics $\left(\mathrm{R}_{0}\right)$, as many of the cosmopolitan species are amfitolerant. Of the steno-ionics species, a large proportion is those acid-neutrophilic $\left(\mathrm{R}_{3-3.5}\right)$ or less acidneutrophilous $\left(\mathrm{R}_{4-4.5}\right)$ ones.

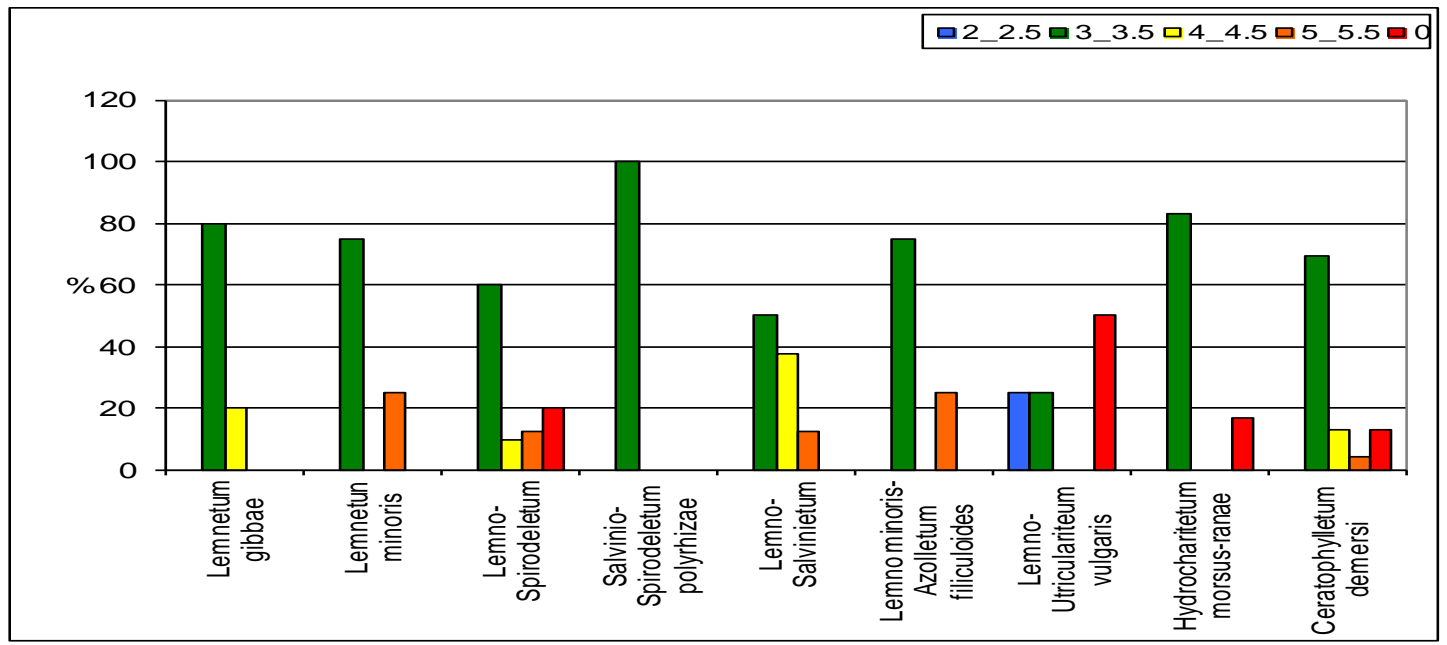

Figure 5: Ecological spectrum for temperature for the communities of the Lemnetea class. 


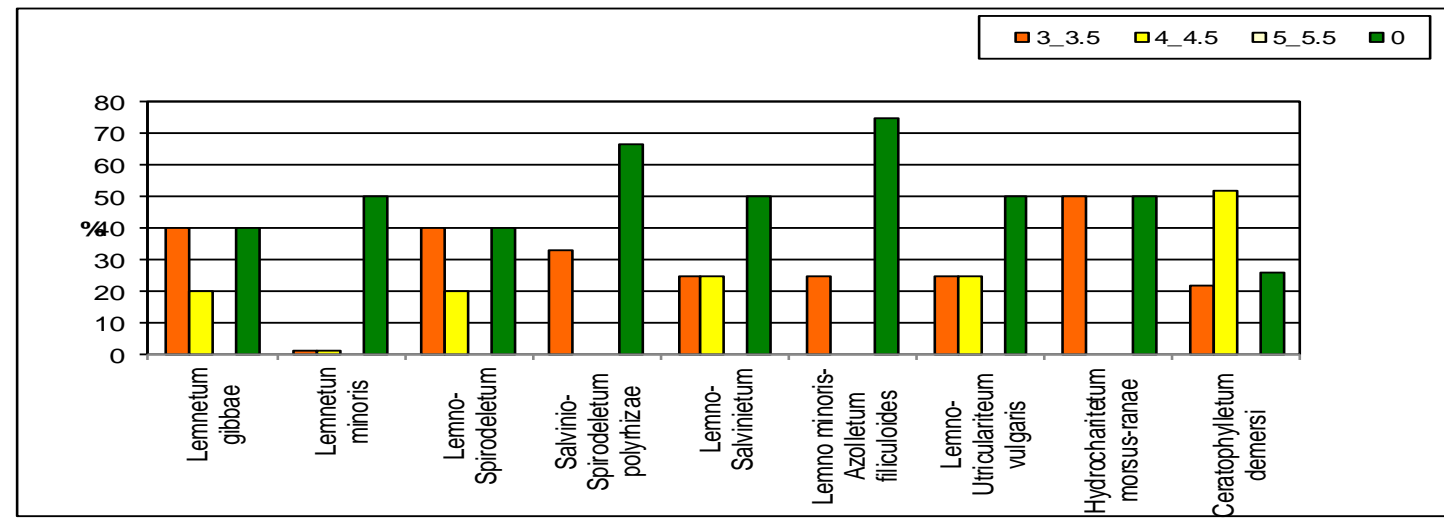

Figure 6: Ecological spectrum for water $\mathrm{pH}$ for the communities of the Lemnetea class.

In terms of social behaviour, the natural pioneer species dominate (NP, with a naturalness value of +3 ), while in the initial stages of the phytocoenogenesis process, with a high reproductive rate, being a species that are tolerant to the extreme environmental conditions, but demanding to nutrient content. Competitive species are well represented (C naturalness value of +5 ), stress-resistant species, especially into those phytocoenosis better knocked off. If phytocoenoses are more stabilized, there are also stress tolerant species, with a broad ecology (generalist species, $G$, with a naturalness value of +4 ) or even specialist species (S, with a naturalness value of +6 ). The latest are met only in the phytocoenosis of the plant community Ceratophylletumdemersi, for which the reisthemost diversified spectrum (Fig. 7). Moreover, they have the most complex structure in the Lemnetea class.

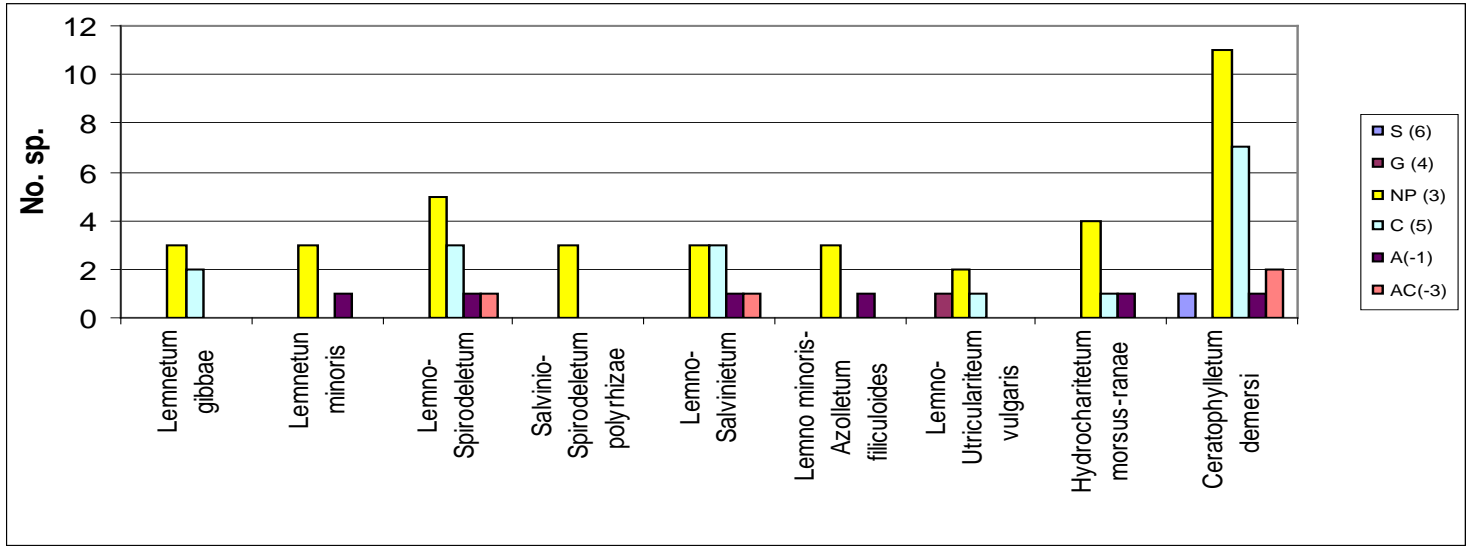

Figure 7: Social behaviour for the communities of the Lemnetea class. 
Table 2: Plant communities from Class Potametea Tx. and Prsg. 1942, Aliance Potamogetonion pectinati (W. Koch 1926) Görs 1977.

\begin{tabular}{|c|c|c|c|c|c|c|c|c|c|c|c|}
\hline Relevé no. & 1 & 2 & 3 & 4 & 5 & 6 & 7 & 8 & 9 & 10 & 11 \\
\hline General coverage (\%) & 9 & 9 & 9 & 9 & 9 & 10 & 9 & 9 & 25 & 6 & 9 \\
\hline Relevé area $\left(\mathrm{m}^{2}\right)$ & 另 & 8 & 8 & 능 & ஜू & $\infty$ & 8 & $\infty$ & 8 & ㅇ & R \\
\hline Relevé code & $\begin{array}{c}\bigcap_{0} \\
\bigcup^{\prime} \\
\underbrace{\prime}_{1}\end{array}$ & $\begin{array}{l}\mathscr{E}_{1} \\
\bigcup^{\prime} \\
\beth_{1}\end{array}$ & $\begin{array}{l}\bar{\sigma}_{1} \\
\bigcup^{\prime} \\
\Xi_{1}\end{array}$ & $\begin{array}{l}\vec{\sigma}_{1} \\
\Xi_{1} \\
\Xi_{1}\end{array}$ & $\begin{array}{c}\vartheta^{2} \\
\bigcup^{\prime} \\
\underbrace{\prime}\end{array}$ & $\begin{array}{l}\beth_{1} \\
\bigcup^{\prime} \\
\beth_{1} \\
n_{1}^{\prime}\end{array}$ & 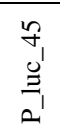 & $\begin{array}{l}\vec{\infty} \\
0^{\prime} \\
\Sigma_{1} \\
\vec{z}\end{array}$ & $\begin{array}{l}\infty^{\prime} \\
0^{\prime} \\
\rho_{1}^{\prime} \\
z^{\prime}\end{array}$ & 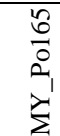 & 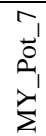 \\
\hline \multicolumn{12}{|l|}{ Potamion } \\
\hline Potamogeton acutifolius & + & + & . & . & . & . & . & . & . & . & . \\
\hline Potamogeton acutifl. f. major & + & + & . & . & . & . & . & . & . & . & . \\
\hline Potamogeton trichoides & . & + & . & + & . & . & . & . & . & . & . \\
\hline \multicolumn{12}{|l|}{ Potametalia and Potametea } \\
\hline Ceratophyllum demersum & 1 & $1-2$ & . & . & + & . & . & + & + & 3 & 2 \\
\hline Najas marina & . & . & . & . & . & . & . & + & . & 1 & . \\
\hline Najas minor & . & . & . & . & . & . & . & . & . & . & . \\
\hline Potamogeton nodosus & . & . & . & + & . & + & 3 & . & . & . & 1 \\
\hline Vallisneria spiralis & . & . & . & . & . & . & . & . & . & . & . \\
\hline Elodea canadensis & . & . & . & . & . & . & . & . & . & . & . \\
\hline Elodea nuttallii & . & . & . & . & + & . & . & . & . & . & + \\
\hline Myriophyllum spicatum & . & . & . & . & . & . & . & 5 & 5 & $3-4$ & 4 \\
\hline Potamogeton crispus & . & . & . & + & . & + & . & . & . & . & + \\
\hline Potamogeton lucens & 5 & 5 & $3-4$ & 4 & 5 & $4-5$ & $3-4$ & . & . & + & . \\
\hline Potamogeton pectinatus & 1 & 1 & + & . & . & . & + & . & + & . & . \\
\hline Potamogeton perfoliatus & . & . & . & + & . & . & . & . & . & + & 2 \\
\hline Potamogeton pusillus & . & . & . & . & + & . & . & +-1 & . & . & + \\
\hline Ranunculus trichophyllus & & ${ }^{2}$ & . & . & . & . & . & . & . & . & . \\
\hline Trapa natans & +-1 & + & + & . & . & . & + & . & . & . & + \\
\hline \multicolumn{12}{|l|}{ Lemnetea } \\
\hline Azolla filiculoides & . & . & . & . & . & . & . & . & . & . & . \\
\hline Lemna minor & + & + & + & + & . & . & + & . & . & . & . \\
\hline Salvinia natans & . & . & . & . & . & . & . & . & . & . & . \\
\hline Spirodela polyrhiza & . & . & + & + & . & . & . & . & . & . & . \\
\hline \multicolumn{12}{|l|}{ Phragmiti-Magnocaricetea } \\
\hline Schoenoplectus lacustris & 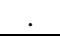 & . & . & . & . & . & . & . & . & . & . \\
\hline \multicolumn{12}{|l|}{ Varyae syntaxa } \\
\hline \multicolumn{12}{|l|}{ Chara foetida } \\
\hline Cladophora glomerata & . & . & . & . & . & . & . & . & . & . & . \\
\hline \multicolumn{12}{|l|}{ Potamion } \\
\hline Potamogeton acutifolius & . & . & . & . & . & + & . & . & . & . & 3 \\
\hline Potamogeton acutifo. f. major & $\dot{\theta}$ & . & . & . & + & . & 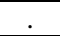 & . & . & . & + \\
\hline Potamogeton trichoides & . & . & . & . & . & + & + & . & . & . & + \\
\hline \multicolumn{12}{|l|}{ Potametalia and Potametea } \\
\hline Ceratophyllum demersum & . & $1-2$ & . & 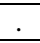 & . & . & . & 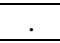 & + & 2 & + \\
\hline Najas marina & . & . & . & 1 & . & + & 3 & 3 & $1-2$ & . & + \\
\hline Najas minor & . & . & . & . & + & 2 & . & . & . & 4 & 3 \\
\hline Potamogeton nodosus & + & 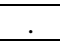 & . & . & . & . & . & $1-2$ & . & + & - \\
\hline Vallisneria spiralis & . & 5 & 4 & 3 & 4 & 2 & . & . & $3-4$ & . & 1 \\
\hline Elodea canadensis & . & . & . & + & 1 & 3 & . & . & . & . & + \\
\hline Elodea nuttallii & & & & & & & & & . & 1 & \\
\hline Myriophyllum spicatum & 4 & + & 2 & 4 & 2 & $1-2$ & 1 & . & + & . & + \\
\hline Potamogeton crispus & & . & . & . & . & . & . & . & . & . &. \\
\hline Potamogeton lucens & & - & . & 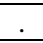 & . & . & 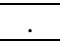 & . & . & . & \\
\hline
\end{tabular}


Table 2 (continued): Plant communities from Class Potametea Tx. and Prsg. 1942, Aliance Potamogetonion pectinati (W. Koch 1926) Görs 1977.

\begin{tabular}{|c|c|c|c|c|c|c|c|c|c|c|c|}
\hline Relevé no. & 1 & 2 & 3 & 4 & 5 & 6 & 7 & 8 & 9 & 10 & 11 \\
\hline General coverage (\%) & 9 & 9 & 9 & 9 & 9 & 10 & 9 & 9 & 25 & 6 & 9 \\
\hline Relevé area $\left(\mathrm{m}^{2}\right)$ & ஜூ & 8 & 8 & 농 & ஜூ & $\infty$ & 8 & $\infty$ & 8 & $\frac{1}{1}$ & 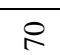 \\
\hline Relevé code & 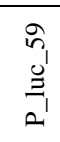 & $\begin{array}{c}8 \\
\oslash_{1} \\
\bigcup^{\prime} \\
\underbrace{}_{1}\end{array}$ & $\begin{array}{l}\overrightarrow{\sigma_{1}} \\
\bigcup^{\prime} \\
\overrightarrow{n^{\prime}}\end{array}$ & $\begin{array}{l}\vec{b} \\
\vec{b}_{1} \\
\sim_{1}\end{array}$ & $\begin{array}{l}\vartheta_{1} \\
\bigcup_{1} \\
D_{1}\end{array}$ & $\begin{array}{l}\beth_{1} \\
\bigcup^{\prime} \\
\exists\end{array}$ & 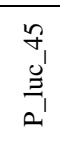 & $\begin{array}{l}\overrightarrow{\infty_{1}} \\
0^{\prime} \\
\sim_{1}^{\prime} \\
\vec{\Sigma}^{\prime}\end{array}$ & $\begin{array}{l}+ \\
\infty_{1} \\
0^{\prime} \\
\sim_{1}^{\prime} \\
\sum^{\prime}\end{array}$ & 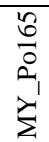 & $\begin{array}{l}\overrightarrow{1} \\
\overrightarrow{0} \\
\vec{\alpha}^{\prime} \\
\vec{\Sigma}\end{array}$ \\
\hline Potamogeton pectinatus & + & 1 & . & . & . &. & . & . & . & . & . \\
\hline Potamogeton perfoliatus & . & . & + & . & . & + & + & . & + & . & . \\
\hline Potamogeton pusillus & . & . & . & . & . & . & . & . & . & . & . \\
\hline Ranunculus trichophyllus & . & . & . & . & . & . & . & . & . & . & . \\
\hline Trapa natans & . & . & . & . & . & . & . & . & . & . & . \\
\hline \multicolumn{12}{|l|}{ Lemnetea } \\
\hline Azolla filiculoides & . & . & . & . & . & . & . & . & . & . & . \\
\hline Lemna minor & . & . & . & . & . & . & . & + & . & . & . \\
\hline Salvinia natans & . & . & . & . & . & . & . & . & . & + & . \\
\hline Spirodela polyrhiza & . & . & . & . & . & . & . & . & . & + & . \\
\hline \multicolumn{12}{|l|}{ Phragmiti-Magnocaricetea } \\
\hline Schoenoplectus lacustris & . & . & . & . & . & . & . & 1 & . & . & . \\
\hline \multicolumn{12}{|l|}{ Varyae syntaxa } \\
\hline Chara foetida & . & . & . & . & . & . & . & . & . & . & . \\
\hline Cladophora glomerata & . & . & . & . & . & . & $1-2$ & . & . & . & . \\
\hline \multicolumn{12}{|l|}{ Potamion } \\
\hline Potamogeton acutifolius & . & . & . & . & . & . & . & . & . & . & . \\
\hline Potamogeton acutif. f. major & . & . & . & . & . & . & . & . & . & . & . \\
\hline Potamogeton trichoides & . & . & . & . & . & . & . & . & . & . & . \\
\hline \multicolumn{12}{|l|}{ Potametalia and Potametea } \\
\hline Ceratophyllum demersum & + & . & . & + & . & . & . & + & $1-2$ & . & . \\
\hline Najas marina & . & . & . & . & . & . & . & . & . & . & . \\
\hline Najas minor & . & . & . & . & . & . & . & . & . & . & . \\
\hline Potamogeton nodosus & . & . & . & +-1 & + & + & . & . & . & 2 & +-1 \\
\hline Vallisneria spiralis & . & . & . & . & . & . & . & . & . & . & . \\
\hline Elodea canadensis & . & . & . & . & . & . & . & . & . & . & . \\
\hline Elodea nuttallii & . & . & . & . & . & . & . & . & . & . & . \\
\hline Myriophyllum spicatum & . & . & . & . & . & . & . & . & . & . & . \\
\hline Potamogeton crispus & . & . & . & . & . & . & . & . & . & . & . \\
\hline Potamogeton lucens & & . & & . & + & . & 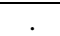 & . & . & . & . \\
\hline Potamogeton pectinatus & $3-4$ & 4 & 4 & 5 & 5 & 5 & 5 & 4 & 4 & 4 & 5 \\
\hline Potamogeton perfoliatus & . & . & . & . & . & . & . & . & . & . & . \\
\hline Potamogeton pusillus & . & . & . & . & . & . & . & . & . & + & . \\
\hline Ranunculus trichophyllus & . & . & . & . & . & . & - & . & . & . & . \\
\hline Trapa natans & 1 & 1 & + & 1 & + & + & +-1 & 1 & $1-2$ & . & . \\
\hline \multicolumn{12}{|l|}{ Lemnetea } \\
\hline Azolla filiculoides & - & . & . & . & . & . & . & . & . & . & . \\
\hline Lemna minor & +-1 & 1 & 1 & + & + & + & + & + & + & + & + \\
\hline Salvinia natans & . & . & . & . & . & . & . & - & . & . & . \\
\hline Spirodela polyrhiza & + & + & + & . & . & + & . & +-1 & . & + & + \\
\hline \multicolumn{12}{|l|}{ Phragmiti-Magnocaricetea } \\
\hline Schoenoplectus lacustris & 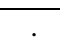 & . & . & . & . & . & . & . & . & . & . \\
\hline \multicolumn{12}{|l|}{ Varyae syntaxa } \\
\hline Chara foetida & $\dot{x}$ & . & . & . & . & . & . & . & . &. & . \\
\hline Cladophora glomerata & & . & & - & & . & 1 & & . & 3 & $1-2$ \\
\hline \multicolumn{12}{|l|}{ Potamion } \\
\hline Potamogeton acutifolius & & . & . & . & . & . & . & . & . & . & . \\
\hline
\end{tabular}


Table 2 (continued): Plant communities from Class Potametea Tx. and Prsg. 1942, Aliance Potamogetonion pectinati (W. Koch 1926) Görs 1977.

\begin{tabular}{|c|c|c|c|c|c|c|c|c|c|c|c|}
\hline \multirow{2}{*}{ Relevé no. } & & & & & & & & & & & \\
\hline & 1 & 2 & 3 & 4 & 5 & 6 & 7 & 8 & 9 & 10 & 11 \\
\hline General coverage (\%) & 9 & 9 & 9 & 9 & 9 & 10 & 9 & 9 & 25 & 6 & 9 \\
\hline Relevé area $\left(\mathrm{m}^{2}\right)$ & 号 & 8 & 8 & 능 & ஜூ & $\infty$ & 8 & $\infty$ & 8 & $R$ & 8 \\
\hline Relevé code & $\begin{array}{l}\Omega_{1} \\
\bigcup^{\prime} \\
\Xi_{1} \\
n^{\prime}\end{array}$ & $\begin{array}{l}\mathscr{\Xi}_{1} \\
\bigcup^{\prime} \\
\Xi \\
D_{1}\end{array}$ & 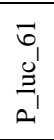 & $\begin{array}{l}\overrightarrow{\sigma_{0}} \\
\Xi_{1} \\
\vec{n}_{1}\end{array}$ & $\begin{array}{l}\mathscr{D}_{1} \\
\bigcup_{1} \\
D_{1}\end{array}$ & $\begin{array}{c}\beth_{1} \\
\bigcup^{\prime} \\
\beth_{1}^{\prime}\end{array}$ & 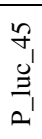 & $\begin{array}{l}\vec{\infty} \\
0^{\prime} \\
\rho^{\prime} \\
z^{\prime}\end{array}$ & $\begin{array}{l} \\
\infty \\
0^{\prime} \\
\Sigma_{1} \\
Z^{\prime}\end{array}$ & $\begin{array}{l}0 \\
0 \\
0 \\
0 \\
\underbrace{\prime} \\
z \\
\Sigma\end{array}$ & 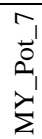 \\
\hline Potamogeton acutif. f. major & . & . & . & . & . & . & . & . & . & . & . \\
\hline Potamogeton trichoides & . & + & . & . & . & . & . & . & . & . & 1 \\
\hline \multicolumn{12}{|l|}{ Potametalia and Potametea } \\
\hline Ceratophyllum demersum & . & . & . & + & + & + & 1 & + & + &. & . \\
\hline Najas marina & . & . & . & . & . & . & . & . & . & . & . \\
\hline Najas minor & . & . & 1 & . & . & . & . & . & . & . & . \\
\hline Potamogeton nodosus & . & . & . & . & . & . & . & + & + & . & . \\
\hline Vallisneria spiralis & . & . & . & . & . & . & . & . & . & . & . \\
\hline Elodea canadensis & . & . & . & . & . & . & . & . & . & . & 5 \\
\hline Elodea nuttallii & . & . & . & . & . & . & . & . & . & . & . \\
\hline Myriophyllum spicatum & 1 & . & . & $1-2$ & . & . & . & . & . & . & 1 \\
\hline Potamogeton crispus & . & . & . & . & . & . & . & . & . & . & . \\
\hline Potamogeton lucens & . & . & . & . & . & . & . & . & . & . & . \\
\hline Potamogeton pectinatus & 4 & 4 & 4 & 5 & . & . & . & 1 & . & + & . \\
\hline Potamogeton perfoliatus & . & . & 1 & . & . & & 2 & + & + & + & . \\
\hline Potamogeton pusillus & . & . & . & + & 5 & $4-5$ & 4 & 5 & $4-5$ & 5 & . \\
\hline Ranunculus trichophyllus & . & +-1 & . & . & . & . & . & . & . & . & . \\
\hline Trapa natans & . & . & . & . & . & . & . & + & . & + & . \\
\hline \multicolumn{12}{|l|}{ Lemnetea } \\
\hline Azolla filiculoides & . & . & . & . & . & . & . & . & . & . & . \\
\hline Lemna minor & . & + & . & . & + & 1 & . & . & . & . & . \\
\hline Salvinia natans & . & + & . & . & . & . & . & . & . & . & . \\
\hline Spirodela polyrhiza & . & + & . & . & + & + & . & . & . & . & . \\
\hline \multicolumn{12}{|l|}{ Phragmiti-Magnocaricetea } \\
\hline Schoenoplectus lacustris & . & . & . & 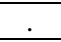 & . & - & . & . & . & 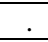 & . \\
\hline \multicolumn{12}{|l|}{ Varyae syntaxa } \\
\hline Chara foetida & + & . & . & . & . & $\dot{\square}$ & . & . & $\dot{\square}$ & . & . \\
\hline Cladophora glomerata & . & . & . & . & . & $2-3$ & . & . & . & . & 3 \\
\hline
\end{tabular}


Table 2 (continued): Plant communities from Class Potametea Tx. and Prsg. 1942, Aliance Potamogetonion pectinati (W. Koch 1926) Görs 1977.

\begin{tabular}{|c|c|c|c|c|c|c|}
\hline \multicolumn{7}{|l|}{ Potamion } \\
\hline Potamogeton acutifolius & . & . & . & . & . & . \\
\hline Potamogeton acutifolius f. major & . & . & . & . & . & . \\
\hline Potamogeton trichoides & + & + & + & . &. & . \\
\hline \multicolumn{7}{|l|}{ Potametalia and Potametea } \\
\hline Ceratophyllum demersum & + & + & . & 2 & . & . \\
\hline Najas marina & + & . & . & + & . & . \\
\hline Najas minor & . & . & . & + & . & . \\
\hline Potamogeton nodosus & . & . & 1 & . & . & . \\
\hline Vallisneria spiralis & + & + & . & . & . & . \\
\hline Elodea canadensis & 5 & 5 & 4 & & . & . \\
\hline Elodea nuttallii & . & . & . & 2 & 4 & 5 \\
\hline Myriophyllum spicatum & + & + & . & . & +-1 & . \\
\hline Potamogeton crispus & . & . & + & . & + & + \\
\hline Potamogeton lucens & . & . & . & . & 2 & . \\
\hline Potamogeton pectinatus & . & . & . & $1-2$ & . & . \\
\hline Potamogeton perfoliatus & . & . & . & . & . & 2 \\
\hline Potamogeton pusillus & . & . & . & . & 1 & . \\
\hline Ranunculus trichophyllus & . & . & . & . & . & . \\
\hline Trapa natans & + & $1-2$ & 3 & + & . & . \\
\hline \multicolumn{7}{|l|}{ Lemnetea } \\
\hline Azolla filiculoides & + & + & . & . & . & . \\
\hline Lemna minor & + & + & + & + & . & . \\
\hline Salvinia natans & + & + & 2 & + & . & . \\
\hline Spirodela polyrhiza & . & . & . & + & . & . \\
\hline \multicolumn{7}{|l|}{ Phragmiti-Magnocaricetea } \\
\hline Schoenoplectus lacustris & . & . & . & . & . & . \\
\hline \multicolumn{7}{|l|}{ Varyae syntaxa } \\
\hline Chara foetida & . & . & . & . & . & . \\
\hline Cladophora glomerata & & & & & & \\
\hline
\end{tabular}

Data and place of relevés: Ass. Potamogetonetum lucentis Hueck 1931: rel. 1-2, 25.06.2012, Şuşca Pond, rel. 3-4, 25.06.2012, Pojejena Pond, rel. 5, 24.06.2012, Sirinia Valley, rel. 6, 23.06.2012, Cerna Valley, rel. 7, 25.06.2012, Divici Pond; Ass. MyriophylloPotamogetonetum lucentis Soó 1934, rel. 8-9, 26.06.2012, Moldova Veche Island, rel. 10, 03.08.2012, Liubcova, rel. 11, 23.06.2012, Vodiţei Valley, rel. 12, 31.07.2012, Moldova Veche Island; ass. Myriophyllo-Potamogetonetum lucentis Soó 1934 sass. vallisnerietosum Coldea et al., 1997, rel. 13-17, 02.08.2012, Nera Pond; Ass. Potamogetonetum pectinati (Hueck 1931) Carstensen 1955, rel. 18-23, 25.06.2012, Divici Pond, rel. 24, 25.06.2012, Belobreşca Pond, rel. 25-26, 24/25.06.2012, Şuşca Pond, rel. 27-28, 25.06.2012, Calinovăţ Isl., rel. 29, 32. 26.06.2012/31.07.2012, Moldova Veche Isl., rel. 30-31, 27.06.2012, Nera Pond; Ass. Potamogetonetum pusilli Soó 1927, rel. 33-34, 25.06.2012, Belobreşca Pond, rel. 35-38, 25.06.2012, Pojejena Pond; Ass. Najadetum marinae Fukarek 1961, rel. 39, 03.08.2012, Liubcova, rel. 40, 31.07.2012, Moldova Veche Island, rel. 41, 02.08.2012, Nera Pond; Ass. Najadetum minoris Ubriszy 1961, rel. 42, 01.08.2012, Şuşca Pond, rel. 43, 02.08.2012, Nera Pond; Ass. Elodeetum canadensis Eggler 1933, rel. 44, 03.08.2012, Cozla, rel. 45-47, 23.09.2012, Moldova Veche Island; Ass. Elodeetum nuttallii Ciocârlan et al., 1997, rel. 48, 01.08.2012, Pojejena, rel. 49, 23.06.2012, Cerna Valley, rel. 50, 23.06.2012, Vodiţei Valley. 
Table 3: Associations from Class Potametea Tx. and Prsg. 1942, Aliance Nymphaeion albae Oberd. 1957.

\begin{tabular}{|c|c|c|c|c|c|c|c|c|c|c|c|c|c|c|c|}
\hline Relevé no. & 1 & 2 & 3 & 4 & 5 & 6 & 7 & 8 & 9 & 10 & 11 & 12 & 13 & 14 & 15 \\
\hline General coverage (\%) & 60 & 100 & 90 & 85 & 95 & 85 & 80 & 90 & 90 & 90 & 100 & 70 & 95 & 90 & 60 \\
\hline Relevé area $\left(\mathrm{m}^{2}\right)$ & 9 & 25 & 9 & 9 & 25 & 9 & 9 & 25 & 25 & 9 & 9 & 9 & 9 & 9 & 9 \\
\hline Relevé code & $\begin{array}{l}\hat{\sigma} \\
\stackrel{1}{n} \\
\vec{z} \\
\vec{z}\end{array}$ & 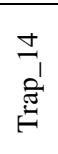 & 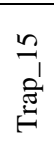 & 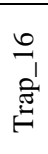 & 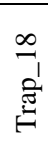 & 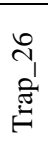 & 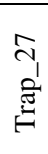 & 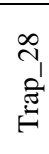 & 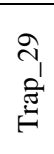 & 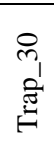 & 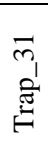 & 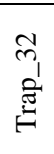 & 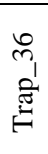 & 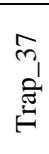 & 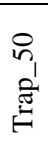 \\
\hline \multicolumn{16}{|l|}{ Potamion } \\
\hline Potamogeton acutifolius & . & . & . & + & . & . & $\cdot$ & . & . & . & . & . & . & . & . \\
\hline $\begin{array}{l}\text { Potamogeton acutifolius f. } \\
\text { major }\end{array}$ & . & . & . & + & . & . & &. & . & . & . & . & . & . & . \\
\hline Potamogeton trichoides & . & . & . & 1 & + & . & . & . & . & . & . & . & . & . & . \\
\hline \multicolumn{16}{|l|}{$\begin{array}{l}\text { Potametalia and } \\
\text { Potametea } \\
\end{array}$} \\
\hline Nymphoides peltata & 3 & 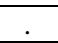 & . & . & . & 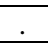 & & & 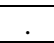 & . & . & 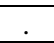 & - & & 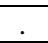 \\
\hline Trapa natans & + & 4 & 5 & $3-4$ & 5 & 5 & 4-5 & $3-4$ & 3 & 5 & 5 & 4 & 5 & 4 & 3-4 \\
\hline Potamogeton natans & . & . & . & . & . & . & . & . & . & . & . & . & . & . & . \\
\hline Potamogeton perfoliatus & . & . & + & . & . & . & . & . & . & . & . & . &. & . & . \\
\hline Najas marina & . & . & . & . & . & . & . & . & . & . & . & . & . & . & . \\
\hline Potamogeton nodosus & + & 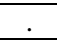 & . & . & . & . & & . & . & . & . & . &. & . & + \\
\hline Ceratophyllum demersum & . & +-1 & 1 & $1-2$ & + & + & + & + & . & . & . & + & . & + & . \\
\hline Elodea canadensis & $1-2$ & . & . & . & . & . & . & . & . & . & . & . & . & . & . \\
\hline Myriophyllum spicatum & + & . & . & . & . & . & . & . & . & . & . & . & . & . & . \\
\hline Potamogeton crispus & . & . & . & + & . & + & . & . & . & . & . & . & . & . & . \\
\hline Potamogeton lucens & . & . & . & . & . & . & 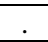 & 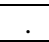 & 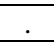 & . & . & . & . & . & - \\
\hline Potamogeton pectinatus & . & + & + & + & . & + & 1 & 2 & $2-3$ & + & + & + & 1 & 1 & $1-2$ \\
\hline Potamogeton pusillus & . & . & . & . & . & . & . & . & . & . & + & . & . & . & . \\
\hline \multicolumn{16}{|l|}{ Elodea nuttallii } \\
\hline Lemnetea & 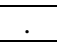 & 2 & 3 & . & + & . & & . & . & . & . & . & . & . & . \\
\hline Azolla filiculoides & + & . & . & . & . & . & 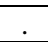 & . & . & . & . & . & . & . & . \\
\hline Salvinia natans & + & . & . & + & . & . & $\dot{\theta}$ & . & . & . & . & . & . & . & . \\
\hline Lemna minor & + & . & . & + & + & 1 & & . & . & . & . & +-1 & +-1 & + & + \\
\hline Lemna gibba & . & . & . & . & . & . & . & . & . & . & . & . & . & . & . \\
\hline Spirodela polyrhiza & + & . & + & + & + & + & & 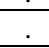 & . & . & . & + & + & + & . \\
\hline \multicolumn{16}{|l|}{ Varyae syntaxa } \\
\hline Chara foetida & . & . & . & . & . & . & & 2 & $1-2$ & . & . & . & . & & . \\
\hline Cladophora glomerata & . & . & . & . & . & . & & . & . & . & . & . & . & & \\
\hline
\end{tabular}


Table 3 (continued): Associations from Class Potametea Tx. and Prsg. 1942, Aliance Nymphaeion albae Oberd. 1957.

\begin{tabular}{|c|c|c|c|c|c|c|c|c|c|c|c|c|c|c|c|c|}
\hline Relevé no. & 1 & 1 & 1 & 1 & 2 & 2 & 2 & 2 & 2 & 2 & 2 & 2 & 2 & 2 & 3 & $\begin{array}{l}3 \\
1\end{array}$ \\
\hline General coverage (\%) & $\begin{array}{l}6 \\
9\end{array}$ & 6 & $\frac{8}{9}$ & $\begin{array}{l}9 \\
6\end{array}$ & $\frac{0}{1}$ & $\frac{1}{1}$ & $\frac{2}{1}$ & $\begin{array}{l}3 \\
1\end{array}$ & $\begin{array}{l}4 \\
8\end{array}$ & $\begin{array}{l}5 \\
6\end{array}$ & $\frac{6}{1}$ & 9 & $\begin{array}{l}0 \\
1\end{array}$ & $\frac{9}{7}$ & 6 & 8 \\
\hline & 5 & 0 & 0 & 0 & $\begin{array}{l}0 \\
0\end{array}$ & $\begin{array}{l}0 \\
0\end{array}$ & $\begin{array}{l}0 \\
0\end{array}$ & $\begin{array}{l}0 \\
0\end{array}$ & 0 & 5 & $\begin{array}{l}0 \\
0\end{array}$ & 0 & $\begin{array}{l}0 \\
0\end{array}$ & 0 & 0 & 0 \\
\hline Relevé area $\left(\mathrm{m}^{2}\right)$ & 9 & 9 & $\begin{array}{l}2 \\
5\end{array}$ & $\begin{array}{l}2 \\
5 \\
\end{array}$ & $\begin{array}{l}2 \\
5\end{array}$ & $\begin{array}{l}2 \\
5 \\
\end{array}$ & $\begin{array}{l}2 \\
5 \\
\end{array}$ & $\begin{array}{l}2 \\
5 \\
\end{array}$ & 9 & $\begin{array}{l}2 \\
5 \\
\end{array}$ & $\begin{array}{l}2 \\
5 \\
\end{array}$ & $\begin{array}{l}2 \\
5\end{array}$ & 9 & 9 & 9 & 9 \\
\hline Relevé code & 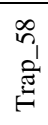 & 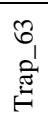 & 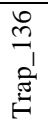 & 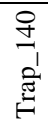 & 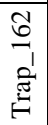 & 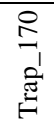 & 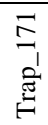 & 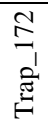 & 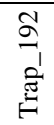 & $\begin{array}{l}\overrightarrow{\vec{m}} \\
\stackrel{\vec{J}}{\vec{\sigma}} \\
\end{array}$ & 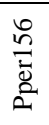 & $\begin{array}{l}\hat{\omega} \\
\stackrel{\vec{\Xi}}{\Xi} \\
\text { م. }\end{array}$ & 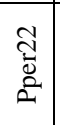 & 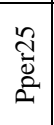 & $\begin{array}{l}\stackrel{Q}{0} \\
\text { ¿ă }\end{array}$ & $\begin{array}{l}\stackrel{\mathscr{N}}{\mathbb{\Xi}} \\
\stackrel{0}{2}\end{array}$ \\
\hline \multicolumn{17}{|l|}{ Potamion } \\
\hline Potamogeton acutifolius & . & . & . & . & . & + & + & . & . & . & . & . & . & . & . & $\dot{\square}$ \\
\hline Potamogeton acutifolius f. major & . & . & . & . & . & + & + & . & . & . & . & . & . & . & . & . \\
\hline Potamogeton trichoides & . & . & . & . & . & . & . & . & . & . & . & . & . & . & + & . \\
\hline \multicolumn{17}{|l|}{ Potametalia and Potametea } \\
\hline Nymphoides peltata & & & & . & & & . & & & . & + & $\cdot$ & . & . & . & $\dot{.}$ \\
\hline Trapa natans & 5 & 5 & 5 & 4 & 5 & 5 & 5 & 5 & + & . & . & . & . & + & + & . \\
\hline Potamogeton natans & . & . & + & . & . & . & . & . & + & 4 & . & . & . & . & . & + \\
\hline Potamogeton perfoliatus & . & + & . & + & . & . & . & . & . & . & 4 & 5 & 5 & 5 & $\begin{array}{c}3- \\
4\end{array}$ & $\begin{array}{c}4- \\
5\end{array}$ \\
\hline Najas marina & . & . & . & + & . & . & . & . & . & 1 & + & . & . & . & . & . \\
\hline Potamogeton nodosus & . & . & . & + & . & . & + & . & . & . & . & . & . & . & $\begin{array}{c}1- \\
2\end{array}$ & . \\
\hline Ceratophyllum demersum & + & 2 & 2 & 2 & + & $\begin{array}{c}1- \\
2\end{array}$ & $\begin{array}{c}1- \\
2\end{array}$ & + & $\begin{array}{l}1- \\
2\end{array}$ & . & 3 & 1 & $\begin{array}{c}1- \\
2\end{array}$ & . & . & . \\
\hline Elodea canadensis & . & . & . & . & . & . & . & . & . & . & . & . & . & . & . & . \\
\hline Myriophyllum spicatum & . & . & . & . & . & - & . & . & + & . & . & . & . & . & . & . \\
\hline Potamogeton crispus & . & . & . & . & . & . & . & . & . & . & . & . & . & . & + & . \\
\hline Potamogeton lucens & . & . & . & . & . & . & . & . & . & . & . & . & . & . & + & \\
\hline Potamogeton pectinatus & + & . & . & + & . & - & . & . & . & . & . & . & 2 & + & . & + \\
\hline Potamogeton pusillus & . & 3 & . & . & . & . & . & . & . & . & . & . & . & . & . & + \\
\hline \multicolumn{17}{|l|}{ Elodea nuttallii } \\
\hline Lemnetea & . & . & . & . & . & + & . & + & . & . & . & . & + & . & . & . \\
\hline Azolla filiculoides & . & . & . & . & . & + & + & . & + & . & + & + & . & . & . & . \\
\hline Salvinia natans & . & . & + & . & . & + & + & . & + & . & + & + & . & . & . &. \\
\hline Lemna minor & + & . & 1 & . & + & 1 & . & 1 & + & . & + & + & . & + & + & - \\
\hline Lemna gibba & & . & & . & 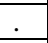 & & + & . & + & . & . & . & . & . & $\cdot$ & . \\
\hline Spirodela polyrhiza & + & . & + & . & + & & + & + & + & . & + & + & . & 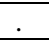 & + & . \\
\hline \multicolumn{17}{|l|}{ Varyae syntaxa } \\
\hline Chara foetida & & & & & & & $\dot{.}$ & & & $\dot{.}$ & & & . & & & \\
\hline Cladophora glomerata & . & . & . & + & . & & . & . & & . & . & . & . & . & . & 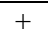 \\
\hline
\end{tabular}

Data and place of relevés: Ass. Nymphoidetum peltatae (All. 1922) Bellot 1951: rel. 1, 23.09.2012, Moldova Veche Island; Ass. Trapetum natantis Kárpáti 1963, rel. 2, 23.06.2012, Orşova, rel. 3, 23.06.2012, Mala Bay, rel. 4, 24.06.2012, Dubova Bay, rel. 5, 23.06.2012, Grăniceri Valley, rel. 6-14, 25.06.2012, Divici Pond, rel. 15, 25.06.2012, Belobreşca Pond, rel. 16 and 19, 25.06.2012/01.08.2012, Şuşca Pond, rel. 17, 25.05.2012, Pojejena Pond, rel. 18, 31.07.2012, Calinovăţ Island, rel. 20, 03.08.2012, Liborajdea, rel. 21-22, 08.04.2012, Dubova, rel. 23, 08.04.2012, Eşelniţa, rel. 24, 23.09.2012, Moldova Veche Island; Ass. Potamogetonetum natantis Soó 1927, rel. 25, 31.07.2012, Moldova Veche Island; Ass. Potamogetonetum perfoliati Miljan 1933, rel. 26-27, 02.08.2012, Nera Pond, rel. 28, 24.06.2012, Tri-Kule, rel. 29, 25.06.2012, Divici Pond, rel. 30-31, 25.06.2012, Pojejena Pond. 
Table 4: Plant communities from Class Potametea R. Tx. and Prsg. 1942, Alliance Ranunculion aquatilis Passarge 1964.

\begin{tabular}{|c|c|c|c|c|c|c|c|c|}
\hline Relevé no. & 1 & 2 & 3 & 4 & 5 & 6 & 7 & 8 \\
\hline General coverage (\%) & 1 & 9 & 9 & 9 & 9 & 9 & 9 & 9 \\
\hline Relevé area $\left(\mathrm{m}^{2}\right)$ & 90 & 80 & 90 & 100 & 90 & 80 & 100 & 70 \\
\hline Relevé code & 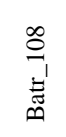 & 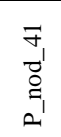 & 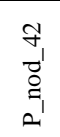 & 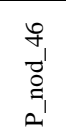 & $\begin{array}{l}g_{1} \\
\sigma_{1} \\
\sigma_{\square} \\
\sigma_{1}\end{array}$ & 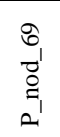 & 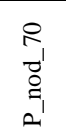 & $\begin{array}{c}\vec{\sigma} \\
\vec{\Xi} \\
\Xi_{1}\end{array}$ \\
\hline \multicolumn{9}{|l|}{ Potamion } \\
\hline Potamogeton obtus. f. latifrons & . & . & . & . & . & . & . & . \\
\hline \multicolumn{9}{|l|}{ Potametalia and Potametea } \\
\hline \multicolumn{9}{|l|}{ Najas marina } \\
\hline Potamogeton nodosus & . & 4 & $3-4$ & 3.4 & 3 & 3 & 3 & 4 \\
\hline Trapa natans & . & + & +-1 & 1 & + & . & . & . \\
\hline Ceratophyllum demersum & . & + & . & . & 2 & . & . & . \\
\hline Myriophyllum spicatum & . & . & . & . & . & . & . & . \\
\hline Potamogeton crispus & . & . & . & . & . & . & . & . \\
\hline Potamogeton lucens & . & . & . & $1-2$ & . & + & . & . \\
\hline Potamogeton pectinatus & . & 1 & $2-3$ & $2-3$ & 2 & 2 & $2-3$ & . \\
\hline Potamogeton perfoliatus & . & . &. & . & . & + & + & + \\
\hline Potamogeton pusillus & . & . & . & . & . & . & . & + \\
\hline Ranunculus trichophyllus & 5 & . & . & . & . & . & . & . \\
\hline Potamogeton trichoides & . & . & . & . & . & + & . & . \\
\hline \multicolumn{9}{|l|}{ Lemnetea } \\
\hline \multicolumn{9}{|l|}{ Azolla filiculoides } \\
\hline Salvinia natans &. & . & . & $\dot{.}$ & . & $\dot{.}$ & $\dot{.}$ & . \\
\hline Lemna minor & + & + & 1 & + & 2 & $1-2$ & + & . \\
\hline Spirodela polyrhiza & + & + & . & . & + & 1 & . & + \\
\hline \multicolumn{9}{|l|}{ Varyae syntaxa } \\
\hline Chara foetida & . & . & . & . & . & . & . & . \\
\hline Cladophora glomerata & . & . & . & . & . & 1 & 1 & 1 \\
\hline \multicolumn{9}{|l|}{ Potamion } \\
\hline Potamogeton obtus.s f. latifrons & . & . & . & . & . & . & + & . \\
\hline \multicolumn{9}{|l|}{ Potametalia and Potametea } \\
\hline Najas marina & . & . & 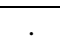 & $1-2$ & . & . & . & . \\
\hline Potamogeton nodosus & 5 & 3 & 3 & $3-4$ & 3 & 4 & 2 & $3-4$ \\
\hline Trapa natans & + & . & . & . & . & . & . & . \\
\hline Ceratophyllum demersum & . & . & . & + & . & . & 2 & 2 \\
\hline Myriophyllum spicatum & . & . & . & . & . & . & . & 1 \\
\hline Potamogeton crispus & . & . & . & . & . & . & + & + \\
\hline Potamogeton lucens & . & . & 2 & . & 1 & . & . & . \\
\hline Potamogeton pectinatus & +-1 & . & 1 & + & + & . & . & . \\
\hline Potamogeton perfoliatus & . & . & . & . & . & . & . & $1-2$ \\
\hline Potamogeton pusillus & . & $2-3$ & . & . & . & . & . & . \\
\hline Ranunculus trichophyllus & . & . & . & . & . & . & . & . \\
\hline Potamogeton trichoides & . & . & . & . & . & . & 1 & . \\
\hline \multicolumn{9}{|l|}{ Lemnetea } \\
\hline Azolla filiculoides & . & . & . & . &. & + & . & . \\
\hline Salvinia natans & . & & . & . & + & + & + & . \\
\hline Lemna minor & + & + & . & . & + & 2 & 3 & . \\
\hline Spirodela polyrhiza & + & + & . & . & + & $1-2$ & $1-2$ & . \\
\hline \multicolumn{9}{|l|}{ Varyae syntaxa } \\
\hline Chara foetida & . & + & . & . & . & . & . & . \\
\hline Cladophora glomerata & . & 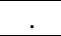 & . & . & . & . & . & \\
\hline
\end{tabular}


Data and place of relevés of table 4: Ass. Ranunculetum (Batrachietum) trichophylli Soó (1927) 1971: rel. 1, 27.06.2012, Nera Pond; Ass. Potamogetonetum nodosi Passarge 1964, rel. 2-4, 25.06.1012, Divici Pond, rel. 5, 25.06.2012, Belobreşca Pond; rel. 6 and 13, 25.06.2012/31.07.2012, Pojejena Pond, rel. 7-11, 25.06.1012, Calinovăţ Island, rel. 12, 31.07.2012, Moldova Veche Island, rel. 14, 02.08.2012, Coronini, rel. 15, 02.08.2012.

Class Potametea pectinati R. Tx. and Prsg. 1942 includes submerged aquatic vegetation or with floating leaves, fixed on substrate, which develop in standing, year-around waters. The vegetation is dominated by perennial species, with communities that tolerate a wide range of water depths. Unless the water levels dry up completely, some species survive periods of low water levels.

One order, three alliances and 14 plant communities (eight associations and one subassociation are newly identified in this protected area) were identified in the "Porţile de Fier” Natural Park (Tabs. 2, 3 and 4).

Over $60 \%$ of the component species belong to the coenotic core, which comprises a decent fraction of these communities in the area, as a consequence of the stable water conditions. This aspect is reflected by distinct clusters in our comparative analysis (Fig. 4).

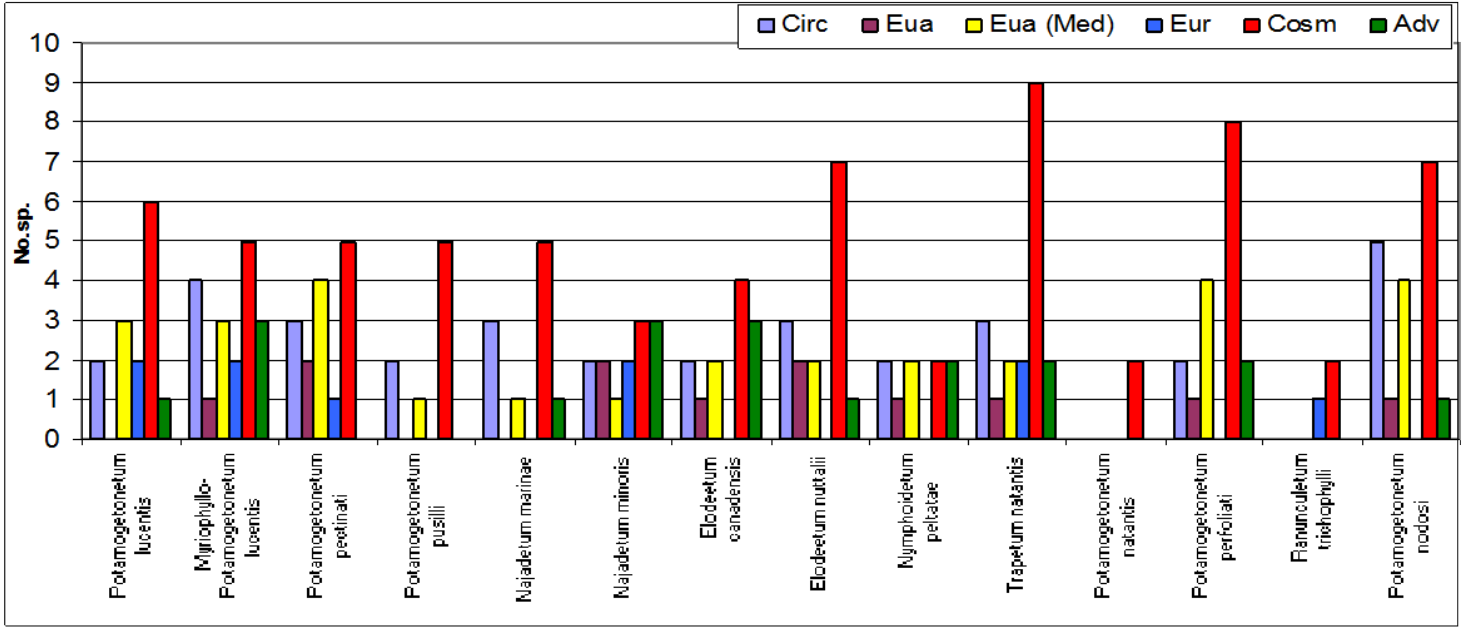

Figure 8: Phytogeografic spectrum for the communities of the Potamogetonetea class.

From a phytogeographical point of view (Fig. 8), the cosmopolitan, circumpolar and the Eurasian species are well represented (especially the Mediterranean ones). Compared with the phytocoenosis of Lemnetea class, the phytogeographical spectrum is more diversified here, as a consequence of a stronger stability of these phytocoenosis. The circumpolar species are present in most communities, benefiting from lower thermal amplitudes of the water. The adventitious species are: Elodea nuttallii, E. canadensis, Vallisneria spiralis, and Azolla filiculoides, the first three species exhibit invasive behavior in the studied area. Communities of Elodea nuttallii replace those of E. canadensis, and also other plant communities edified by indigenous species. Only few communities are devoid of invasive species, e.g. Potametum pectinati (occurs more frequently at the fringes of ponds along the Danube River, where the water current is faster), Potametum pusilli, Potametum natantis and Ranunculetum (Batrachietum) trichophylli. The last two communities are rare in the area. 


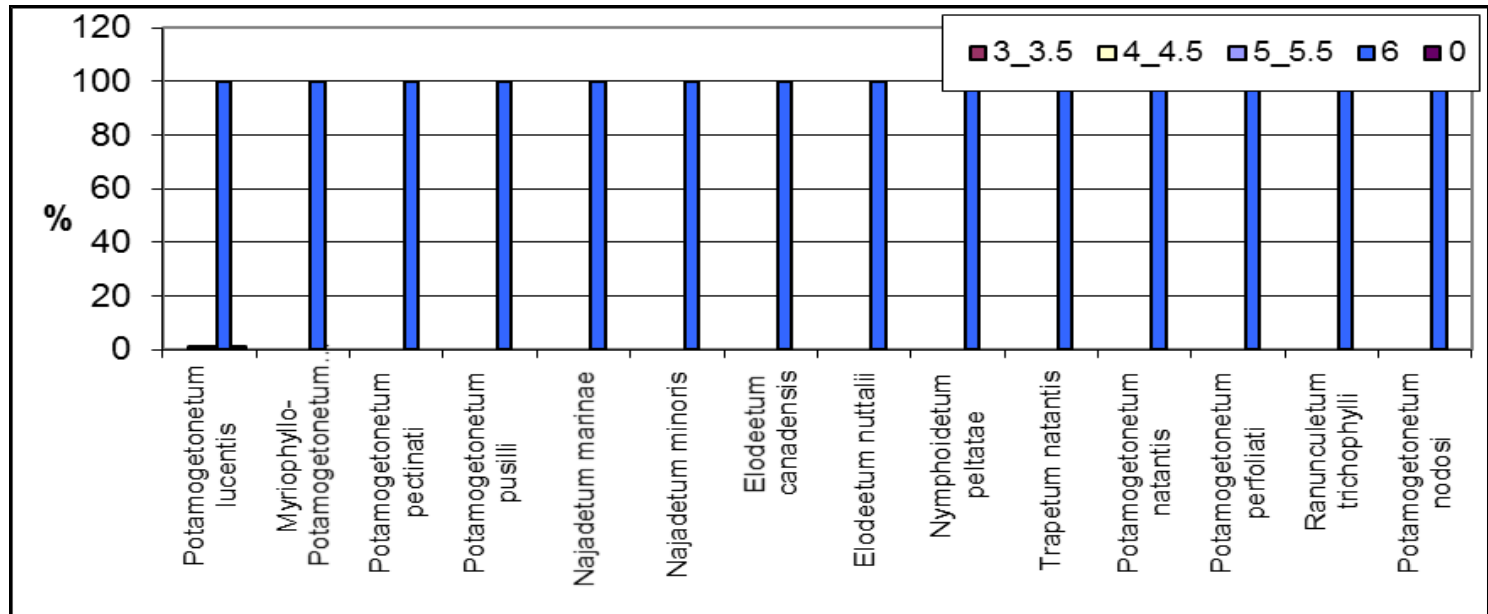

Figure 9: Ecological spectrum for humidity for the communities of the Potamogetonetea class.

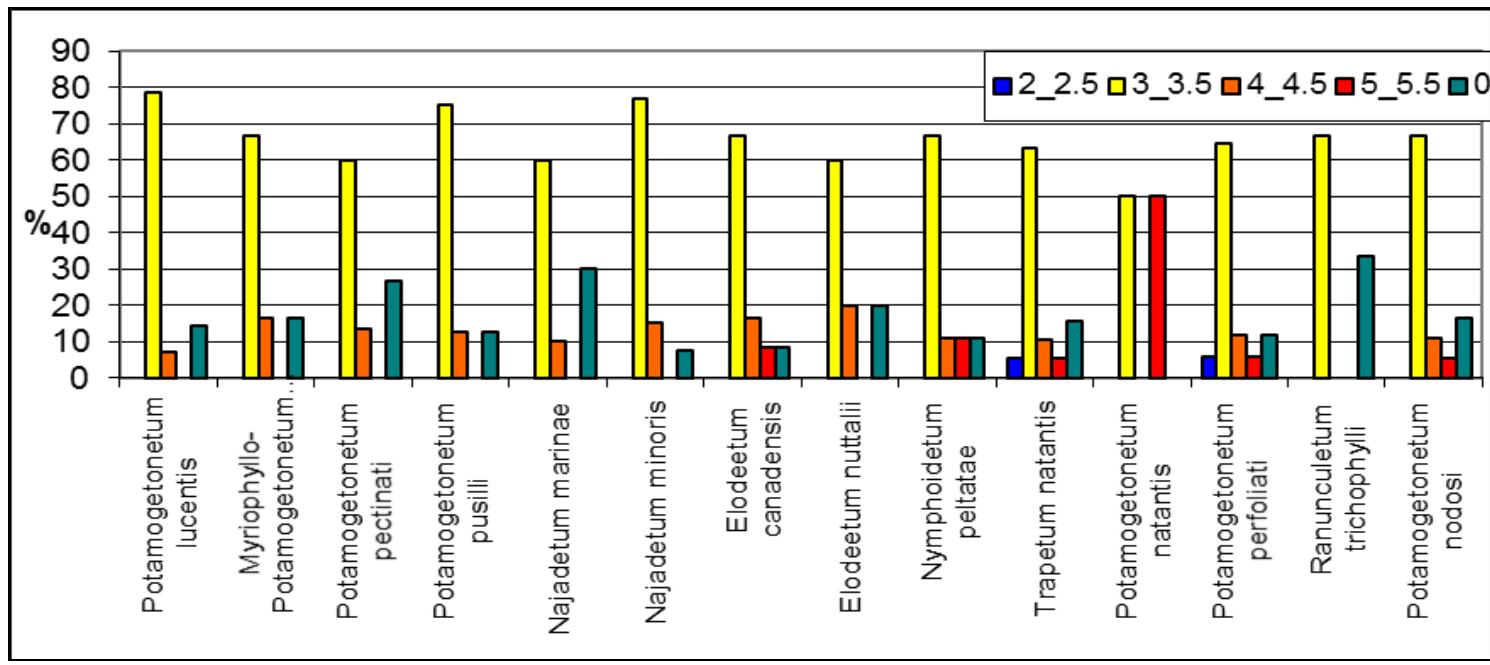

Figure 10: Ecological spectrum for temperature for the communities of the Potamogetonetea class.

The ecological spectra, related to humidity (Fig. 9) and temperature (Fig. 10) are similar to those of the Lemnetea class, prevailing hydrophilous $\left(\mathrm{U}_{6}\right)$ and the micromesothermal species $\left(\mathrm{T}_{3-3.5}\right)$; with a good occurrence of the moderate thermophilous species ( $\left.\mathrm{T}_{4-4.5}\right)$ and thermophilous ( $\left.\mathrm{T}_{5-5.5}\right)$ as a result of Mediterranean climate. Compared to water $\mathrm{pH}$, among the steno-ionics species, the dominant are the weak acid-neutrophilous species $\left(\mathrm{R}_{4-4.5}\right)$, followed by acid-neutrophilous $\left(\mathrm{R}_{3-3.5}\right)$. The euri-ionics species $\left(\mathrm{R}_{0}\right)$, show a permanent disturbance of the habitat (Fig. 11). 


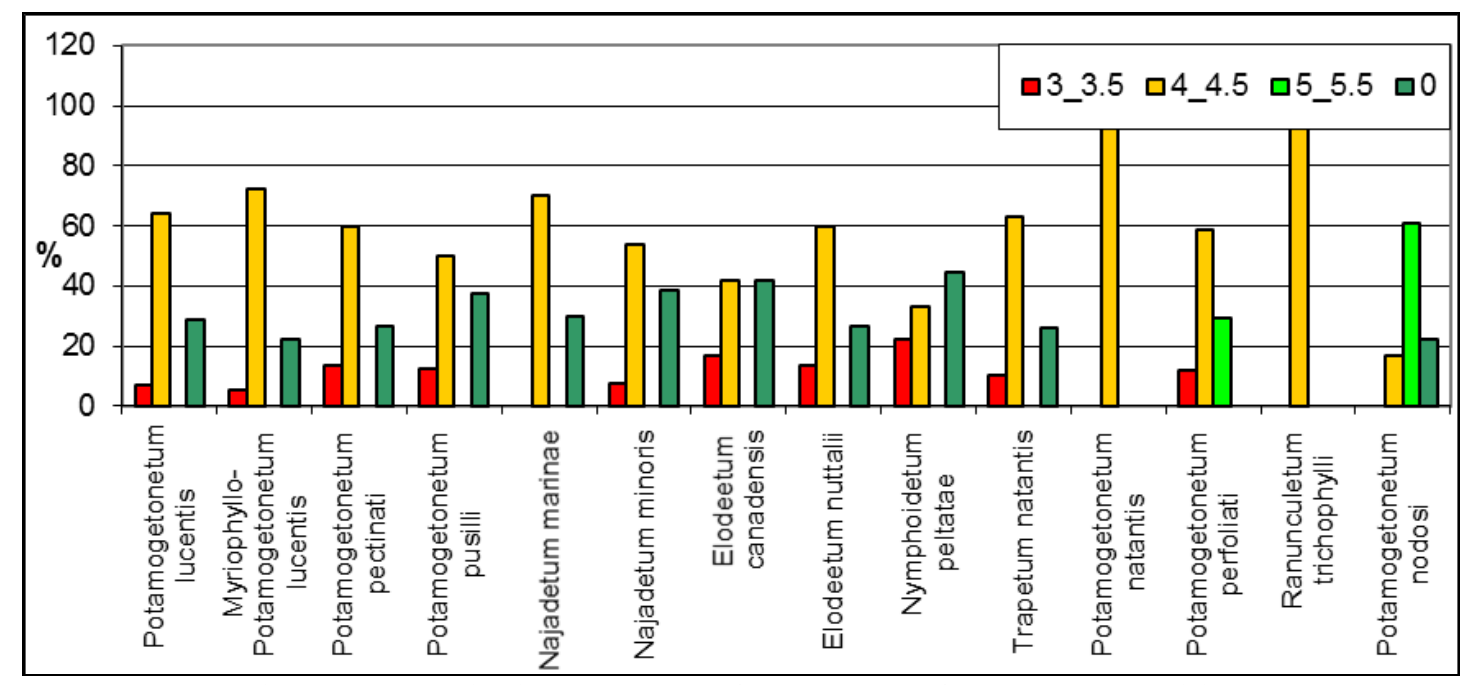

Figure 11: Ecological spectrum for water $\mathrm{pH}$ for the communities of the Potamogetonetea class.

In terms of social behaviour, the competitive species dominate ( $\mathrm{C}$ with a naturalness value of +5 ), being stress-resistant species, are more present in a cohesive phytocoenosis. Natural pioneers are well represented (NP, with a naturalness value of +3 ), especially by those plant communities located in areas with strong currents (Potamogetonetum lucentis) or by the recently installed (Najadetum marinae). In the case of stabilized phytocoenoses, stress-tolerant plant species are also present, with a broad ecology (generalist $G$, with a naturalness value of $+4)$. The presence of species with limited ecology (S, with a naturalness value of +6 ), in almost all plant communities is noteworthy, which indicates a constant, stable feature of phytocoenosis, a harmonized structure of the aquatic habitats with extreme conditions, a structure that was accomplished over time (Fig. 12). Potamogetonetum natantis and Ranunculetum trichophylli plant communities are exceptions, seldom appear in the investigated area. The plant communities of Ranunculion aquatilis (aquatic vegetation developed in shallow waters, smoothly flowing and sometimes temporarily) are in competition with large aquatic macrophytes, which is a reason why they are becoming fewer and fewer in southeastern Europe (http://www.sci.muni.cz/botany/vegsci/vegetace.php?lang=en\&typ=VBD).

The characteristic species of this alliance is Ranunculus trichophyllus. It was identified in a single phytocoenosis only, in the mini delta of Nera, and only in June, during the flooding period. Two alien invasive species, Elodea canadensis and E. nuttallii, were recorded (Borhidi, 1995), but according to other references, Vallisneria spiralis is also an invasive plant species in Europe (Săvulescu, 1952-1976). 


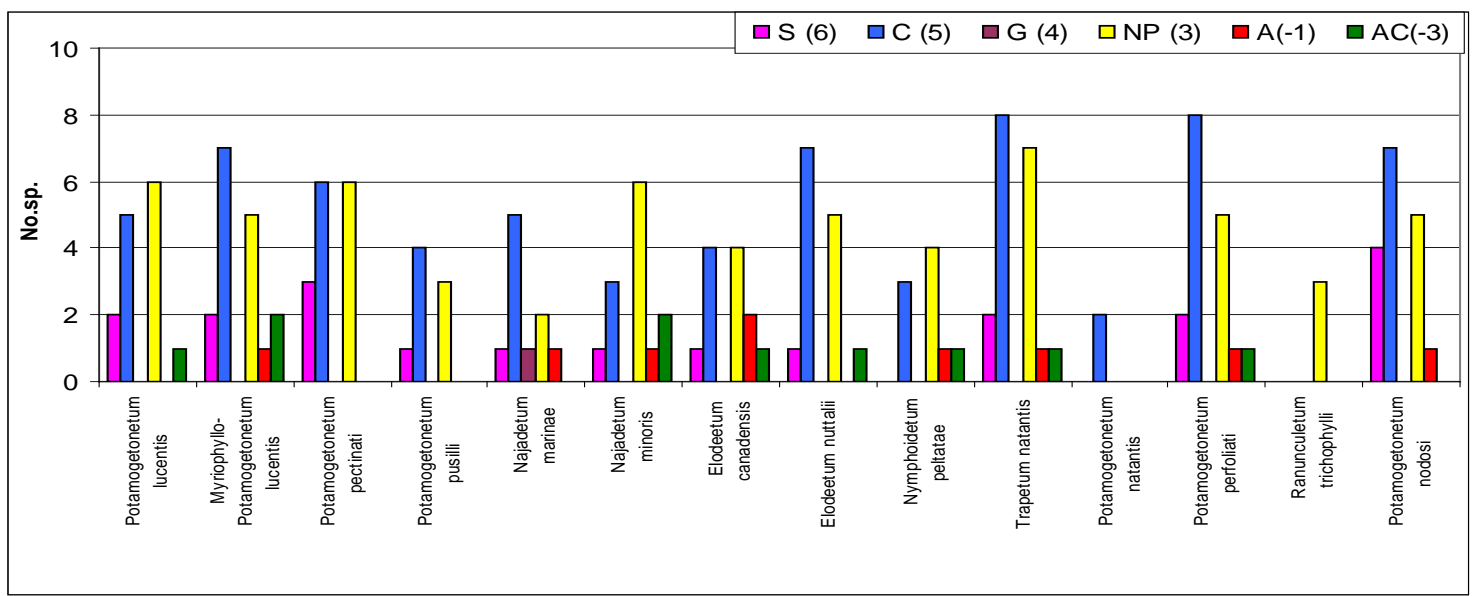

Figure 12: Social behaviour spectrum for the associations of the Potamogetonetea class.

\section{Anthropogenic impact on natural habitats}

Based on phytosociologic relevés and field investigations, there have been identified the following three types of natural habitats, namely: $\mathbf{3 1 5 0}$ - Natural eutrophic lakes with vegetation of Magnopotamion or Hydrocharition, 3160 - Natural dystrophic lakes and ponds, and 3260 - Water courses lowland to mountain level, with vegetation from Ranunculion fluitantis and Callitricho-Batrachion. The habitat 3160 is the most widespread in the area, especially represented by Trapetum natantis plant communities, which cover large areas of the ponds, shallow waters and small bays. The massive development of this plant community is detrimental to other submerged or floating communities, up until September, when the area covered by this habitat is halved, as long as the dominant species begins to decompose. This situation, led to some difficulties during summer time, water crafts are slowed in their movement along the ponds; furthermore, this plant community slow the development of the fish fauna because of the low amount of oxygen in the water; on the other hand, it has a positive effect on birds, giving them peace, bringing environmental services by water denitrification (Tall et al., 2011), especially considering, where the terrain includes croplands.

These habitats host protected or threatened plant species, including those on the red lists, like: Azolla filiculoides (Dihoru and Negrean, 2009), Potamogeton trichoides (Oltean et al., 1994), Salvinia natans (Bern Convention, 1979; Dihoru and Negrean, 2009), Elodea canadensis (Boşcaiu et al., 1994), Najas marina (Boşcaiu et al., 1994) (in our opinion, it is a mistake to include in a red list/red book, those species which are aliens in a certain region, e.g. Azolla filiculoides or Elodea canadensis in Europe).

The plant communities of these habitats are dominated by natural pioneer species (especially floating species) and competitive species (mostly submerged species), as a result of habitat disturbance through seasonal water fluctuation. According to Zohary and Ostrovsky (2011), increasing water level affects the habitat diversity, species richness and abundance and favour the income and proliferation of allochtone generalists, with negative response in species richness. Some of the alien species have an invasive feature in natural habitats. Elodea canadensis, Vallisneria spiralis, and especially Elodea nuttallii, which eliminate other plants, represent a real problem. On the other hand, Vallisneria spiralis grows abundantly in Nera Pond, taking out the communities edified by Najas marina and Najas minor. 
A permanent anthropogenic pressure is exerted on aquatic and swamp habitats from the "Porţile de Fier" Natural Park. The development of tourism, as an alternative to the economic downturn in the studied area, is reflected by a growing number of buildings (as hotels, holiday cottages, pontoon bridges, etc.), but mostly by leasing the banks of the Danube River to private persons or companies. All these lead to a decrease of the cover of spontaneous riparian vegetation and water eutrophication. In addition, each year, the Danube River and its tributaries, bring a significant amount of waste, the most affected area in this regard being the Nera Delta. This area is grazed by sheep, while the isle of Moldova Veche is grazed by horses, thus leading to a higher eutrophication. The answer of plant communities toward eutrophication is obvious through a higher representation of euri-ionic plant species.

\section{CONCLUSIONS}

The aquatic vegetation of the "Porţile de Fier" Natural Park has a mosaic-type appearance, mainly determined by the speed of water flow, and by the topography of the studied area. Thus, all the identified associations are concentrated in the area of Divici, Belobreşca, Şuşca, Pojejena, and Nera ponds, the golfs between Orşova and Dubova, and associated ponds and bays of the island of Calinovăţ and Moldova Nouă. Most of the plant communities have been recorded in the area of the Moldova Nouă Isle and Nera Pond, which require a special attention, as part of the integrated management of the Natura 2000 sites: "ROSPA0026 Cursul Dunării - Baziaş-Porţile de Fier" and "ROSCI0206 Porţile de Fier”.

As a result of this study, eleven plant associations, and a sub-association, are newlyidentified in the investigated area. The presence of all of these plant communities could be a consequence of vegetation succession after the construction of the dam. An increase of wealth of the aquatic communities involves diversification of the algal and invertebrate fauna components, as some authors have already concluded (Dudley, 1988; Melo et al., 2002; Takeda et al., 2003; Thorp et al., 1997). The structure of these plant communities is influenced by the flow regime, level fluctuation and nutrient concentration in the water.

While developing the management plan should take into account the surveillance of the spreading of invasive plants (especially Elodea canadensis, Elodea nuttallii and Valisneria spiralis) and a mechanical removal of them. On the other hand, opening up corridors through the aquatic vegetation and allowing a controlled eco-tourism, with small speed boats, is an occupational alternative in this area sought by tourists, while the mines were closed and fishing is strongly restricted in the area of the "Porţile de Fier" Natural Park.

\section{ACKNOWLEDGEMENTS}

Financial support for this work was generously provided by the LIFE10 NAT/RO/740 project - Improving the conservation status for the priority species and habitats in the "Iron Gates” wetlands. 


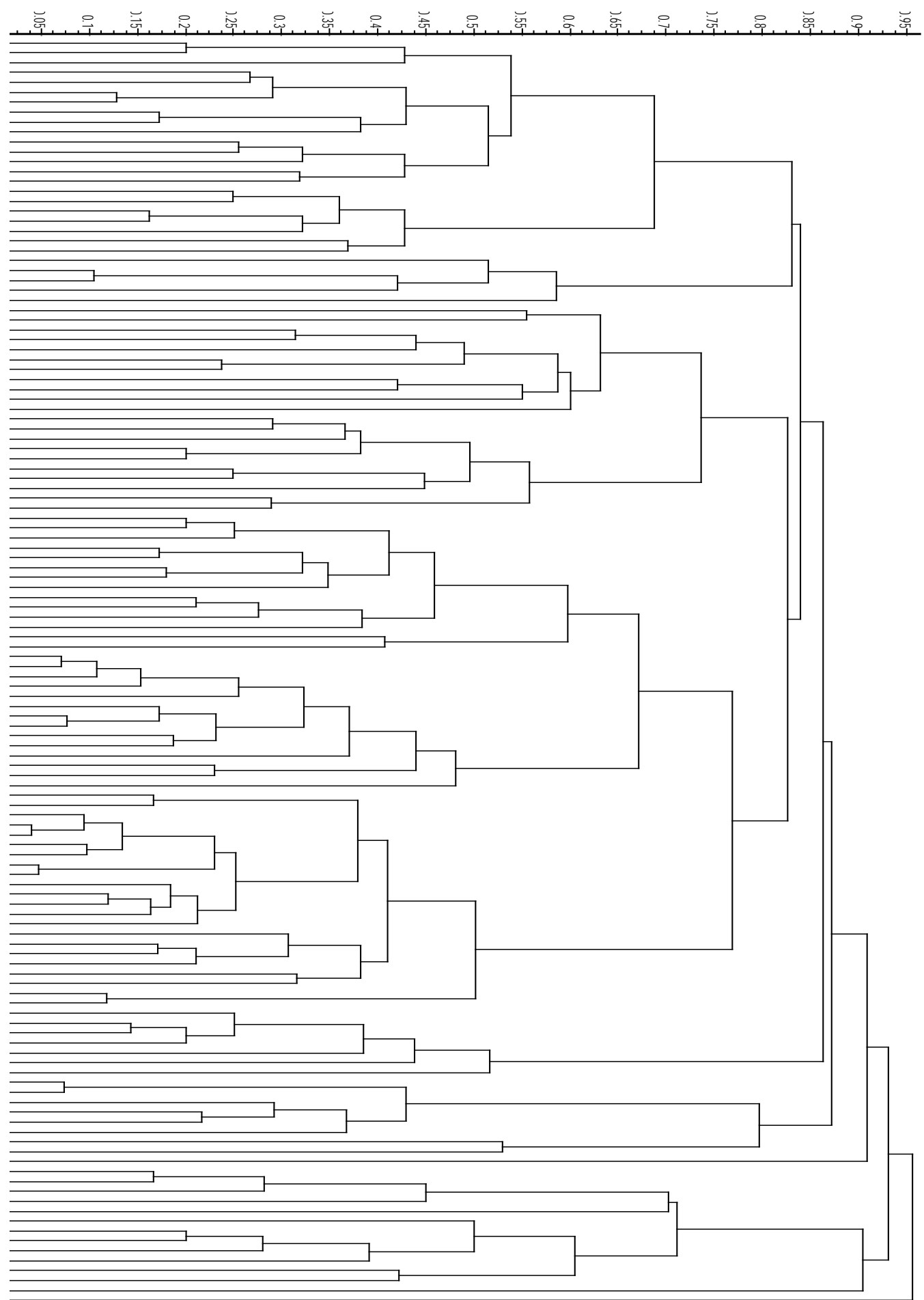

Figure 12: Aquatic communities’ dendrogram (UPGMA, Bray Curtis Index). 


\section{REFERENCES}

1. Bell S. S., McCoy E. D. and Mushinsky H. R., 1991 - Habitat structure: the physical arrangement of objects in space, Chapman and Hall, London.

2. Blaustein L. and Schwartz S. S., 2001 - Why study ecology in temporary pools? Israel Journal of Zoology, 47, 303-312.

3. Borhidi A., 1995 - Social behaviour types, their naturalness and relative ecological indicator values of the higher plants of the Hungarian flora, Acta Botanica Hungarica, 39, 97-182.

4. Borza A., 1947-1949 - Conspectus Florae Romaniae, Regionumque affinum, I-II, Tipografia „Cartea Românească”, Cluj, 360.

5. Boşcaiu N., Coldea G. and Horeanu C., 1994 - Lista roşie a plantelor vasculare dispărute şi rare din flora României, Ocrotirea Naturii și Mediului Înconjurător, 38, 1, 45-46. (in Romanian)

6. Braun-Blanquet J., 1964 - Pflanzensoziologie, Springer Verlag, Berlin. (in German)

7. Brönmark C. and Hansson L. A., 2010 - The Biology of lakes and pounds, Second edition, Oxford University Press, 285.

8. Călinescu R., Coteţ P., Florea N., Iancu M., Martiniuc C., Savu A., Sârcu I. and Stoenescu Ş., 1955 - Geografia fizică a R. P. Române, Bucureşti. (in Romanian)

9. Călinescu R. and Iana S., 1964 - Consideraţiuni biogeografice asupra Defileului Dunării, Analele Universităţii Bucureşti, Ştiințe Naturale, 12, 1, 151-167. (in Romanian)

10. Cărăuş I., 2012 - Algae of Romania, A distributional checklist of actual algae, (Version 2.3), http://www.seaweedafrica.org/pdf/562DF96D13b4823893SWjO3A7893/50442.pdf, 809.

11. Ciocârlan V., Doniţă N. and Turcu G., 1969 - Contribuţii floristice din defileul Dunării, sectorul Cozla-Berzasca, Studii şi Cercetări de Biologie, Seria Botanică, 21, 3, 205-208. (in Romanian)

12. Ciocârlan V., 2000 - Flora ilustrată a României, Pteridophyta et Spermatophyta, Bucureşti, Edit. Ceres, 1138. (in Romanian)

13. Coldea G., 1991 - Prodrome des associations végétales des Carpates du sud-est (Carpathes Roumaines), Documents in Phytosociology, Camerino, 13, 317-539. (in French)

14. Coldea G. (ed.), Sanda V., Popescu A. and Ştefan N., 1997 - Les associations végétales de Roumanie, 1, Les associations herbacées naturelles, Cluj-Napoca, 261. (in French)

15. Cristea V., 1991 - Fitosociologie şi vegetaţia României, Îndrumător de lucrări practice, Universitatea „Babeş-Bolyai”, Cluj-Napoca. (in Romanian)

16. Cristea V., 1993 - Fitosociologie şi vegetaţia României, Universitatea „Babeş-Bolyai”, ClujNapoca, 314. (in Romanian)

17. Cristea V., Gafta D. and Pedrotti F., 2004 - Fitosociologie, Edit. Presa Universitară Clujeană, Cluj-Napoca, 394. (in Romanian)

18. Csürös Ş., Pop I., Hodişan I. and Csürös-Káptalan M., 1968 - Cercetări floristice şi de vegetaţie între Orşova şi Eşelniţa, Contribuții Botanice, 277-312. (in Romanian)

19. Dihoru G., Cristurean I. and Andrei M., 1973 - Vegetaţia dintre Valea Mraconiei - Depresiunea Dubova, Acta Botanica Horti București, 1972-1973, 353-423. (in Romanian)

20. Dihoru G. and Negrean G., 2009 - Cartea roşie a plantelor vasculare din România, Edit. Academiei Române, Bucureşti, 630. (in Romanian)

21. Doniţă N., Popescu A., Paucă-Comănescu M., Mihăilescu S. and Biriş I. A., 2005 - Habitatele din România, Edit. Tehnică Silvică, Bucureşti, 496. (in Romanian)

22. Doniţă N., Popescu A., Paucă-Comănescu M., Mihăilescu S. and Biriş I. A., 2006 - Habitatele din România, Edit. Tehnică Silvică, Bucureşti, 56. (in Romanian)

23. Dudley T. L., 1988 - The role of plant complexity and epiphyton in colonization of macrophytes by stream insects, Verhandlungen der Internationalen Vereinigung für Limnologie, 23, 1, 1153-8. 
24. Gafta D., Mountford J. O. (eds), Alexiu V., Anastasiu P., Bărbos M., Burescu P., Coldea G., Drăgulescu C., Făgăraş M., Gafta D., Goia I., Groza G., Micu D., Mihăilescu S., Moldovan O., Nicolin L. A., Niculescu M., Oprea A., Oroian S., Paucă Comănescu M., Sârbu I. and Şuteu A., 2008 - Handbook for interpretation of Natura 2000 habitats from România, Cluj-Napoca, 101.

25. Glăvan V. and Geanana M., 1972 - Solurile, Atlasul complex „Porţile de Fier”, Edit. Academiei Române, 142. (in Romanian)

26. Grecescu D., 1898 - Conspectul Florei României, Bucureşti. (in Romanian)

27. Grigore S. and Coste I., 1978 - Cercetări asupra vegetaţiei dintre Moldova Veche şi Pescari, Studii și Cercetări de Geologie, Geografie, Biologie, Reşiţa, 7, 173-189. (in Romanian)

28. Heuffel J., 1858 - Enumeratio Plantarum in Banatu Temesiensi, Vindabonae.

29. Liţescu S., Pascale G. and Sârbu A., 2003 - Aquatic flora and vegetation of semiflowing lakes Porţile de Fier I and Porţile de Fier II, Studii și Cercetări de Biologie, 8, 60-65, Bacău.

30. Matacă S. Ş., 2002 - Conspectul sistematic al plantelor vasculare din Parcul Natural Porţile de Fier (I), Drobeta, 11-12, 255-295. (in Romanian)

31. Matacă S. Ş., 2005 - Parcul Natural Porţile de Fier, Floră, vegetaţie şi protecţia naturii, Edit. Universitaria, Craiova, 550. (in Romanian)

32. Melo S. M., Takeda A. M. and Monkolski A., 2002 - Seasonal dynamics of Callibaetis willineri (Ephemeroptera, Baetidae) associated with Eichornia azurea (Pontederiaceae) in Guaraná Lake of the upper Paraná River, Brazil, Hydrobiologia, 470, 1-3, 57-62.

33. Morariu I., Danciu M. and Ularu P., 1969 - Date noi din flora Porţilor de Fier, Studii și Cercetări de Biologie, Seria Botanică, 21, 1, 17-22. (in Romanian)

34. Morariu I. and Danciu M., 1970 - In der Gegend der Eisernen Tores (Porţile de Fier) gemeldete Pflanzenassoziationen, Revue Roumaine de Biologie - Botanique, 15, 5, 419-429. (in German)

35. Oltean M., Negrean G., Popescu A., Roman N., Dihoru G., Sanda V. and Mihăilescu S., 1994 Lista roşie a plantelor superioare din România, in Oltean M. (ed.) Studii, sinteze, documentaţii de ecologie, Academia Română - Institutul de Biologie, 1, 52, Bucureşti. (in Romanian)

36. Păun M., Cîrţu M., Cîrţu D. and Popescu G., 1968 - Materiale pentru flora dintre Berzeasca şi Pescari, județul Caraş-Severin, Buletinul Ştiințiific, 10, 47-62, Craiova. (in Romanian)

37. Podani J., 1993 - SYN-TAX-PC, Computer Programs for mulivariate data analysis in ecology and systematics, version 5.0 - User's guide, Scientia Publication, Budapest.

38. Popescu A. and Ştefureac T., 1976 - Vegetations forschungen aus dem Sektor Sviniţa-Trikule Eisernes tor Rumäniens, Acta botanica Horti Bucurestiensis, 1975-1976, 341-368. (in German)

39. Popescu A., Sanda V. and Oroian S., 1997 - Vegetaţia Deltei Dunării, Marisia, 25, 3-126.

40. Popescu A. and Sanda V., 1998 - Conspectul florei cormofitelor spontane din România, Acta botanica Horti Bucurestiensis, 336. (in Romanian)

41. Popp N., 1971 - Principalele probleme de geografie fizică în opera lui Vâlsan G., Opere alese, Bucureşti, Edit. Ştiinţifică, 38-69 (in Romanian)

42. Raclaru P. and Alexan M., 1972 - Flora Defileului Dunării, Baziaş-Pojejena, Analele Universității Bucureşti, Biologie vegetală, 21, 201-217. (in Romanian)

43. Raclaru P. and Alexan M., 1973 - Asociaţii vegetale palustre din Defileul Dunării, Baziaş-Pojejena, Studii și Cercetări de Biologie, Seria Botanică, 25, 2, 131-139. (in Romanian) 
44. Rai U. N. and Sinha S., 2001 - Distribution of metals in aquatic edible plants: Trapa natans (Roxb.), Environmental Monitoring and Assesment, 79, 241-252.

45. Resmeriţă I., Vicol E. C., Coldea G. and Schneider E., 1972 - Cartarea vegetaţiei din sectorul Valea Eşelnița, Studii și Cercetări de Biologie, Seria Botanică, 24, 3, 213-220. (in Romanian)

46. Roman N., 1971 - Elemente noi pentru caracterizarea fitogeografică a Porţilor de Fier, Studii şi Cercetări de Biologie, Seria Botanică, 23, 6, 477-484. (in Romanian)

47. Rooke J. B., 1984 - The invertebrate fauna of four macrophytes in a lotic system, Freshwater Biology, 14, 507-513.

48. Sanda V., Şerbănescu G. and Zăvoianu I., 1968 - Aspecte ale florei şi vegetaţiei palustre din Clisura Cazanelor, Studii și Cercetări de Biologie, Seria Botanică, 20, 3, 217-224. (in Romanian)

49. Sanda V., Şerbănescu G., Raclaru P. and Alexan M., 1970 - Contribuţii la cunoaşterea staţiunilor cu Acorus calamus L., Studii și Cercetări de Biologie, Seria Botanică, 22, 6, 481-489.

50. Sanda V., Popescu A. and Doltu M. I., 1980 - Cenotaxonomia și corologia grupărilor vegetale din România, Studii și Comunicări, Ştiințe Naturale, Sibiu, 24 (supl.), 1-172. (in Romanian)

51. Sanda V., Popescu A., Doltu M. I. and Doniţă N., 1983 - Caracterizarea ecologică şi fitocenologică a speciilor spontane din flora României, Studii și Comunicări, Ştiințe Naturale, Sibiu, 25 (supl.), 1-126. (in Romanian)

52. Sanda V., Popescu A. and Coldea G., 1994 - Asociaţiile vegetale din clasa Lemnetea minoris R. Tx. 1955 din România, Contribuții Botanice, 1993-1994, 5-11. (in Romanian)

53. Saunders D. L. and Kalff J., 2001 - Nitrogen retention in wetlands, lakes and rivers, Hydrobiologia, 443, 205-212.

54. Săvulescu T., (coord.), 1952-1976 - Flora Republicii Populare Române - Flora Republicii Socialiste România, I-XIII, Edit. Academiei Române. (in Romanian)

55. Sârbu A., Smarandache D., Janauer G. and Pascale G., 2006 - Elodea nuttallii (Planchon) St. John - a competitive hydrophyte in the Romanian Danube River corridors, Proceedings 36th International Conference of IAD, 4-8 September, Vienna.

56. Sârbu A., Janauer G., Schmidt-Mumm U., Filzmoser P., Smarandache D. and Pascale G., 2011 Characterisation of the potamal Danube River and the Delta: connectivity determines indicative macrophyte assemblages, Hydrobiologia, 671, 75-93.

57. Srivastava S., Yadav A. and Shukla D. N., 2014 - Effects of lead and its phytoremediation potential in Trapa natans L., Asian Journal of Research in Chemistry, 4, 434.

58. Şerbănescu G. and Sanda V., 1970 - Cercetări asupra vegetaţiei de luncă şi dealuri între Cazanele Mari şi Plavişeviţa, Studii și Cercetări de Biologie, 22, 3, 171-178. (in Romanian)

59. Ştefureac T., 1970 - Cercetări asupra florei şi vegetaţiei de la Porţile de Fier, Analele Universității Bucureşti, Biologie Vegetală, 19, 193-204. (in Romanian)

60. Ştefureac T., Popescu A., Zitti R. and Mihai G., 1971 - Analiza cormofitelor din sectorul Şviniţa-Tricule (Clisura Dunării), Comunicări Botanice, 12, 111-131. (in Romanian)

61. Takeda A. M., Souza-Franco G. M., Melo S. M. and Monkolski A., 2003 - Invertebrados associados a macrófitas, in Thomaz S. M. and Bini L. M. (eds), Ecologia e Manejo de Macrófitas Aquáticas, 243-260, Maringá, Eduem. (in Portuguese)

62. Tall L., Caraco N. and Maranger R., 2011 - Denitrification hot spots: dominant role of invasive macrophyte Trapa natans in removing nitrogen, Ecological Applications, 21, 8, 31043114.

63. Tetelea C., 2005 - Potenţialul geoecologic al ecosistemelor acvatice din Parcul Natural Porţile de Fier, Rezumat teză doctorat, Universitatea din Bucureşti. (in Romanian) 
64. Thorp A. G., Jones R. C. and Kelso D. P., 1997 - A comparison of water-column macroinvertebrate communities in beds of different submersed aquatic vegetation in the tidal freshwater Potomac River, Estuaries, 20, 1, 86-95.

65. Todor I., Gergely I. and Bârcă C., 1971 - Contribuţii la cunoaşterea florei şi vegetaţiei din zona Defileului Dunării, Contribuții Botanice, 203-256. (in Romanian)

66. Tutin T. G., Heywwood V. H., Burges N. A., Moore D. M., Valentine D. H., Walters S. M. and Webb D. A., 1964-1980 - Flora Europaea, Cambridge: Cambridge University Press, 1-5.

67. Vivian-Smith G., 1997 - Microtopographic heterogeneity and floristic diversity in experimental wetland communities, Journal of Ecology, 85, 71-82.

68. Williams P., Whitfield M., Biggs J., Bray S., Fox G., Pascale N. and Sear D., 2003 Comparative biodiversity of rivers, streams, ditches and ponds in an agricultural landscape in Southern England, Biological Conservation, 115, 329-341. 